\title{
les premiers temps de la mécanique des sols
}

\author{
par le Professeur \\ A. W. Skempton \\ Imperial College - Université de Londres \\ Ancien Président de la Société Internationale \\ de Mécanique des sols et des Travaux de Fondation \\ (Traduction Armand Mayer)
}

\section{Introduction}

La mécanique des sols moderne est née pendant les années 1910 à 1930 , en grande partie à la suite des travaux de Terzaghi (Bjerrum, Casagrande, Peck et Skempton, 1960) et de Fellenius ainsi que des collègues de celui-ci en Suède (Bjerrum et Flodin, 1960). Le terme de mécanique des sols a été utilisé d'une façon générale après une série d'articles publiés par Terzaghi dans l'Engineering News Record (Terzaghi, 1925).

Cependant ce sujet d'études existait, sinon sous ce nom, bien avant 1925, même si les éléments n'en avaient pas été rassemblés et reconnus comme une discipline cohérente. II existait plutôt comme une série de sujets distincts, la théorie des pressions de terrain, la connaissance pratique des glissements des terrains argileux, etc., avec peu de liaison entre les observations en place et l'analyse théorique et surtout sans le principe essentiel de la contrainte effective. Néanmoins des travaux importants théoriques et pratiques ont été faits en mécanique du sol à cette époque et c'est sur les plus importants de ceux-ci que je porterai mon attention au cours de la présente conférence.

Parmi les travaux des anciens, le célèbre essai de Coulomb de 1773 a été reproduit et traduit en anglais avec une discussion très approfondie par Hayman (1972); l'ouvrage de Collin sur les glissements des sols argileux a été traduit (Shriever, 1956) avec un commentaire biographique et analytique par moimême; Rowe (1969) a rendu compte des essais d'Osborne Reynolds sur la dilatance et le traité de Boussinesq de 1885 a été réimprimé en 1969 avec une préface de Caquot. Kérisel a fait en 1956 une excellente récapitulation des travaux français anciens, Feld a donné en 1921 un résumé des essais de pressions de terrain, tandis que Flodin et Broms (1977) ont rassemblé des renseignements sur l'histoire des problèmes géotechniques dans les argiles molles. Quant à l'aspect pratique de ces questions, des études excellentes ont été publiées par Peck (1948) sur les

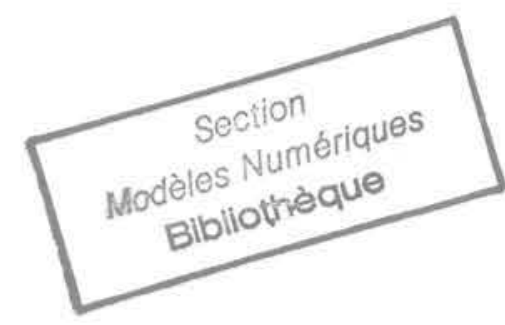

fondations d'immeubles à Chicago, par Glossop $(1960,1961)$ sur les injections, en 1976 sur l'emploi de l'air comprimé dans la construction des tunnels et des puits. Mais parmi les 30 sujets qui seront examinés dans cet exposé, certains sont très peu connus et tous ont fait l'objet d'un nouvel examen. Lors de sa publication, l'exposé oral sera complété par de courtes notes biographiques.

\section{I - Première phase}

Différentes tentatives pour traiter des problèmes de mécanique des sols ont été faites avant Coulomb dans ce que l'on peut appeler la période pré-classique et l'on peut trouver maints exemples de travaux de fondation et digues en terre dessinés rationnellement et d'une conception saine.

\section{GAUTIER - 1717}

Dissertation sur l'épaisseur des Culées des Ponts... sur l'Effort et la Pesanteur des Arches... et sur les Profils de Maçonnerie qui doivent supporter des chaussées, des Terrasses et des Remparts.

Paris - Cailleau - 1717

Dans son chapitre sur les murs de soutènement. Gautier indique que les remblais peuvent être exécutés (en sable, en terre ou en glaise) et il a mesuré la pente naturelle des sols des deux premières catégories en mesurant l'angle qui correspond à la stabilité des matériaux déversés. II donne $5 / 3\left(31^{\circ}\right)$ pour un sable propre et sec et $1 / 1\left(45^{\circ}\right)$ pour une terre qui vient d'être extraite et pulvérisée. II n'a pas fait d'essais sur de l'argile, mais il indique que quand elle est bien tassée 
elle exerce des pressions moindres que la terre ou le sable sur des murs de soutènement. II donne aussi les poids volumiques ci-après : eau 63 , sable 116 , terre 84 , maçonnerie 126 , le tout en livres par pied cube. (10; 18,$4 ; 13,3 ; 20 \mathrm{kN} / \mathrm{m}^{3}$ )

Dans son exposé d'une théorie des murs de soutènement, il commence par remarquer que du fait qu'un massif de terre tient en équilibre à son angle de talus naturel, le rôle du mur est simplement de tenir le coin de terre situé au-dessus de ce talus (fig. 1). Admettant que le mur a été construit sur une certaine hauteur $C E$, la hauteur de terre au-dessus de ce niveau tiendrait à son angle de talus naturel FG. Elle devrait donc avoir une base $E F$ où $E F=\frac{1}{2} B E$. Bien entendu la pente JG serait stable si le triangle EFJ était remplacé par de la maçonnerie. Ce raisonnement est valable pour un niveau quelconque entre $\mathrm{C}$ et $\mathrm{B}$, c'est-à-dire que le profil théorique pour un mur serait un triangle $B C D$ où I'on aurait $C D=\frac{1}{2} B C$. En pratique le mur doit avoir une certaine épaisseur et il est en général construit avec une pente amont de $1 / 5: 1$. Comme règle simple on peut admettre que le mur doit être construit avec cette pente amont et la même section que le triangle BCD. Autrement dit l'épaisseur moyenne du mur devrait être le quart de sa hauteur.

Gautier donne un tableau des dimensions des murs et applique exactement la règle ci-dessus pour un mur de 20 pieds de haut $(6 \mathrm{~m})$. Pour des hauteurs inférieures, il augmente l'épaisseur pour compenser l'effet, proportionnellement plus grand, des charges mobiles en surface, du remplissage et, ce qui ne paraît pas logique, il admet une faible réduction du rapport ci-dessus, pour les murs de plus grande hauteur.

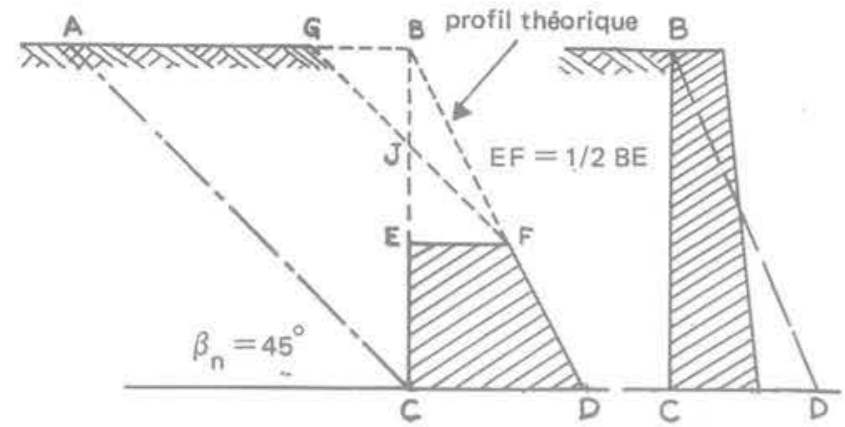

si $B C=20 \mathrm{ft}$, l'épaisseur moyenne du mur est $5 \mathrm{ft}$ pour $\beta_{n}=45^{\circ}$

Fig. 1 Gautier, 1717

\section{Biographie}

Henri Gautier (1660-1737), après avoir été ingénieur au service du roi dans les Travaux Maritimes, a été chargé de hautes fonctions dans le Corps des Ponts et Chaussées lors de la création de celui-ci en 1715. Son traité de la Construction des Chemins (Paris, 1715) et son Traité des Ponts (Paris, 1716) étaient des manuels bien connus. (Nouvelle Biographie Générale, 1857.)

\section{BÉLIDOR - 1729}

La Science des Ingénieurs dans la conduite des Travaux de Fortifications et d'Architecture Civile.

Paris - Jombert - 1729

Dans son traité bien connu destiné aux ingénieurs civils et militaires, Bélidor s'étend longuement sur l'étude des murs de soutènement. II admet la classification des remblais de Gautier et prend pour les calculs types le cas de la terre avec un angle de talus naturel de $45^{\circ}$. Si la terre n'avait pas de frottement interne la poussée sur un mur vertical avec un remblai horizontal serait égale au poids du triangle $A B C$ (fig. 1). Mais les terrains naturels ont une certaine cohésion (ténacité) et il est raisonnable de prendre pour la poussée la moitié du poids. C'est-à-dire que Bélidor admet que :

$$
\mathrm{P}_{\mathrm{B}}=\frac{1}{2} \gamma \frac{\mathrm{H}^{2}}{2} \text { ou } \quad \mathrm{K}_{\mathrm{o}}=0,5
$$

et il montre que la résultante passe par le tiers inférieur du mur. II néglige le frottement de la terre contre le mur.

II a étendu l'analyse au cas des murs supportant un remblai incliné et des murs comportant des contreforts amont ou une face amont inclinée. Le principe du calcul est d'équilibrer le moment de la poussée des terres et du poids du mur autour de son pied, en prenant la densité de la maçonnerie égale à $1,5 \gamma$ et ensuite de majorer l'épaisseur du mur de $25 \%$. II indique que ce coefficient de sécurité est particulièrement utile dans le cas de murs de quai ou de murs supportant une route.

Pour les murs à face amont verticale et face aval inclinée à 1/5:1 l'épaisseur moyenne (y compris la majoration de $25 \%$ ) est de $1 / 3$ de la hauteur si le terrain retenu est horizontal. Bélidor se rend parfaitement compte que cette disposition implique une fondation satisfaisante. Si le terrain n'est pas résistant il suggère d'utiliser une fondation sur pieux.

On peut indiquer que différentes tentatives pour résoudre le problème des poussées de terrain ont été faites au cours des 40 années qui ont suivi, mais sans comporter de grands progrès par rapport à Bélidor. $\mathrm{Ce}$ travail a fait l'objet d'un examen complet par Mayniel (1808).

\section{Biographie}

Bernard Forest de Bélidor (1671-1761), professeur de mathématiques au collège militaire de La Fère, a pris du service actif dans l'arme du génie pendant la guerre de Succession d'Autriche et ensuite exerça à Paris les fonctions de Général de Brigade. II devint membre de l'Académie des Sciences et de la Société Royale Britannique. Son ouvrage sur "l'Architecture Hydraulique" (4 vol. Paris 1737-1753) a constitué la base des connaissances des ingénieurs civils français et britanniques jusqu'au début du $19^{e}$ siècle. (Dictionnaire des biographies scientifiques, 1970.) 


\section{GRUNDY - 1766}

Etudes et évaluations pour une digue en terre à Grimsthorpe dans le Lincolnshire. Manuscrit pp. 143-151. Examen... Rapports... et Évaluations de travaux de drainage, de navigation et autres études de la profession d'Ingénieur, par John Grundy de Spalding, Ingénieur. (Bibliothèque de I'Institution des Ingénieurs Civils.)

En 1748, un lac de grande dimension, d'une étendue de 8 hectares, a été constitué pour orner la propriété du Duc d'Ancaster à Grimsthorpe par la construction d'une digue en terre. Étudiée par John Grundy, cette digue (qui existe encore) avait une hauteur de 18 pieds $(5,5 \mathrm{~m})$ et comportait un noyau central en argile, ainsi qu'il ressort d'un rapport datant de 1758 . II semble que l'on n'ait conservé aucun projet original, mais en 1766 Grundy a fait un projet avec une coupe pour une digue à construire en aval, qui aurait ajouté 20 acres ( 5 ha) au lac. Le projet et la coupe ne sont pas datés, mais il existe un rapport sur le projet daté de 1766 et un plan, dessiné par Grundy en 1767, montrant le lac existant et l'emplacement de la nouvelle digue, telle qu'elle était projetée (voir G.M. Binnie, Transactions de la Sté Newcomen - 47 (1976) page 212).

La digue devait avoir une hauteur maxima de 25 pieds $(7,5 \mathrm{~m})$ une pente amont de $3,5 / 1$, une pente aval de $2 / 1$, une largeur en crête de 20 pieds $(6 \mathrm{~m})$ et un noyau central d'argile de 6 pieds $(2 \mathrm{~m})$ de large. Les talus devaient être constitués par de la terre compactée, obtenue à partir des matériaux disponibles sur place. II était spécifié que le noyau devait être de l'argile bien humidifiée et compactée après avoir été homogénéisée. Cette argile devait être prélevée à un emplacement choisi à une certaine distance du site. Grundy admet un tassement de 2 inches par pied $(1 / 6)$ et indique que la digue au moment de sa construction devait être de 29 pieds $(9 \mathrm{~m})$.

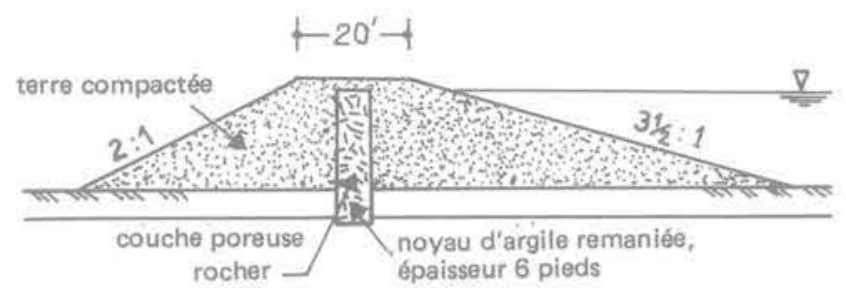

hauteur maxima du barrage : 25 pieds

tassement prévu : 2 pouces par pied

Fig. 2 Projet de barrage en terre par John Grundy, 1766

Telles sont les plus anciennes indications connues sur la construction d'une digue en terre en Angleterre et elles s'appliquent probablement avec de faibles différences au barrage construit en 1748. Une disposition particulièrement intéressante est la présence d'un noyau d'argile.

Des noyaux analogues ont été utilisés dans des barrages construits par des projecteurs de canaux à la fin du $18^{\mathrm{e}}$ siècle et les noyaux d'argile malaxée, en général à talus inclinés, ont été une disposition standard dans les barrages de retenue du $19^{\circ}$ siècle.
La coupe et les spécifications de Grundy sont un bon exemple de projet rationnel basé sur l'expérience, l'observation et le sens critique de l'ingénieur, sans application de calculs, en un temps où le calcul était ou non-existant ou insuffisant pour pouvoir être appliqué.

\section{Biographie}

John Grundy (1719-1783), un ingénieur conseil bien connu, exécuta surtout des travaux de drainage et de navigabilité dans l'Est et le Nord-Est de l'Angleterre. (A.W. Skempton et Esther Wright - Les premiers membres de la Société Smeatonienne des Ingénieurs Civils (Trans. de la Soc. Newcomen - 44 (1971) pp. 23-47.)

\section{PERRONET - 1769}

Mémoire sur l'éboulement qui arrive quelquefois à des portions de montagnes et autres terrains élevés, et sur les moyens de prévenir ces éboulements et de s'en garantir dans plusieurs circonstances.

Paris - 1769 - Réimprimé (pp. 631-643) dans la deuxième édition des œuvres de M. Perronet. Paris - Didot - 1788.

Ce mémoire de 12 pages de Perronet amorce l'étude de la stabilité des pentes.

Les pentes peuvent exister dans les terrains naturels (terre vierge) ou les remblais. Les pentes naturelles qui sont restées stables pendant une longue période le resteront sauf si des changements sont apportés à leur état. Ces changements peuvent correspondre à une surcharge de la partie supérieure de la pente par un remblai ou une construction lourde, à une excavation au pied de la pente ou à des inflitrations d'eau qui en réduiront la résistance.

Dans des terrains intacts, résistants, on peut découper des parois verticales (Perronet vise sans doute des excavations temporaires), mais dans les sols mous et les sables secs, les pentes, même constituées par des matériaux intacts, adopteront une inclinaison d'environ $30^{\circ}$ sur I'horizontale.

Les remblais constitués par une terre excavée depuis un certain temps et qui a perdu une grande partie de sa cohésion, ou une terre qui vient d'être remaniée et qui a encore moins de cohésion, tiendront sous un angle d'environ $35^{\circ}$ pour les plus résistants (et non $45^{\circ}$ comme il est communément admis) à environ $30^{\circ}$ pour les sables et la terre molle, exactement comme pour des pentes naturelles et jusqu'à $18^{\circ}$ ou même moins pour des argiles humides. Par contre les gros graviers et les rochers concassés peuvent tenir sous un angle de $40^{\circ}$ à $45^{\circ}$.

Ces angles correspondent à des talus de hauteur modérée, dont les pentes sont à peu près planes. Dans les talus de grande hauteur, les pentes tendent à être concaves et à avoir une inclinaison moindre que celle qui a été indiquée ci-dessus. A titre d'exemple on peut citer le remblai d'enrochements de la Vallée des Bois de la Haie, sur la route Paris-Nancy, qui a une hauteur de 142 pieds $(43 \mathrm{~m})$ et une inclinaison de $1,5 / 1$ de la crête au pied mais un profil concave avec un creux de 6 pieds 8 pouces ( 2 mètres). 
Avant de faire un déblai dans une zone vallonnée il est opportun de déterminer la nature du terrain par des sondages, des trous à la tarière ou des puits, de manière à extraire des échantillons; et si l'on trouve des couches d'argiles inclinées dans la direction du déblai que I'on se propose d'exécuter, l'ingénieur ne devrait pas hésiter à rechercher un tracé moins dangereux.

Car, dans ce cas, des glissements peuvent se produire, même sur des pentes très faibles. De plus, la pente peut devenir instable même si l'on ne l'entaille pas, si elle s'imprègne d'eau qui réduit le frottement interne. Un bon drainage est alors nécessaire et Perronet décrit rapidement deux exemples, à Marly en 1758 et près de Croix Fontaine en 1765 où l'exécution d'un drainage a stabilisé des glissements de terrain sur des lits d'argile de manière efficace.

\section{On peut ajouter quatre remarques :}

1 La première édition des œuvres de M. Perronet a été publiée en in-folio; deux volumes en $1782-83$ et un troisième en 1789. La seconde édition de 1788 a le texte complet en in-quarto avec un atlas in-folio de plans et dessins.

2 Le mémoire original imprimé sur les Éboulements (à la Bibliothèque Nationale) est daté du 5 juillet 1769. II a été réimprimé en 1788 et aussi dans le troisième tome de ses œuvres en 1789, mais sans la date. Je remercie le Professeur Kerisel de m'avoir envoyé des photocopies du mémoire de 1769 et d'une version manuscrite (également datée) provenant de la Bibliothèque de l'École des Ponts et Chaussées. Le manuscrit comporte huit dessins annexés.

3 II a èté dit souvent que Perronet, dans ce mémoire, avait été le premier à décrire les surfaces de glissement incurvées. En réalité il se rapporte seulement à la surface concave de la pente elle-même. Ceci résulte clairement du texte et est démontré par l'un des dessins auxquels il est fait allusion ci-dessous.

4 Comme nous l'apprend le mémoire de Perronet sur les fondations sur pieux (imprimé dans les volumes de 1782 et 1788), des échantillons avaient été prélevés avec un tube en fer de 2 pouces de diamètre battu dans le sol. A un pied de distance tout le long de la tige ce tube avait des orifices ou des poches, inclinées vers le bas, avec une languette se projetant de la base; celles-ci retenaient lors de l'extraction du tube de petits échantillons de terrain.

\section{Biographie}

Jean-Rodolphe Perronet (1708-1794) - Ingénieur en Chef des Ponts et Chaussées et Directeur de l'Ecole pendant 47 ans depuis la création de celle-ci en 1747 jusqu'à sa mort. Il était membre de l'Académie des Sciences et de la Société Royale Britannique et le plus éminent ingénieur civil de France du $18^{\circ}$ siècle; il était notamment connu pour ses ponts dont le Pont de Neuilly, celui de Pont Ste-Maxence et le Pont de la Concorde - (Dictionnaire des biographies scientifiques), 1975.

\section{II - La Mécanique des Sols classique (1776-1845)}

Cette section traite de la méthode d'analyse des équilibres limites de Coulomb en mécanique des sols, des développements ultérieurs et de queiques essais de pression de terrain.

\section{COULOMB - 1776}

Essai sur une application des règles de maximis et minimis à quelques problèmes de statique relatifs à l'architecture.

Mémoires de l'Académie Royale des Sciences, vol. 7 (1776) pp. 343-382.

Coulomb a présenté sa communication à l'Académie les 10 mars et 2 avril 1773. Elle fit l'objet d'un rapport par Bossut et Borda un an plus tard et fut publiee en 1776. Cette communication, qui constituait une contribution d'importance fondamentale dans la science de l'ingénieur civil, traitait de la résistance au cisaillement de la maçonnerie et des sols, des pressions de terrain, de la stabilité des voûtes et de la résistance des poutres. Les points principaux en mécanique des sols sont les suivants :

1 Coulomb introduit la notion que la résistance au cisaillement que peut développer une surface a de maçonnerie ou de sol, sur laquelle agit une force normale $\mathrm{N}$, est la somme de la cohésion du matériau et d'une résistance au frottement. Elle s'exprime par l'expression

$$
S=c a+\frac{1}{n} N
$$

où c est la cohésion (sans direction) par unité de surface et $\frac{1}{n}$ le coefficient de frottement interne.

2 II introduit également la recherche de la surface de glissement critique en utilisant l'équation (1) ci-dessus qui permet d'obtenir la poussée maxima sur un mur de soutènement ou la résistance à la compression minima d'une colonne. En général la surface de glissement telle qu'elle résulte du cisaillement est incurvée, mais dans le problème qui est traité dans ce rapport elle est assimilée à un plan pour simplifier.

3. II montre ensuite que la résistance à la compression d'une colonne verticale de faible hauteur et de section droite $\mathrm{A}$ est

$$
Q=\frac{c A}{\cos \alpha\left(\sin \alpha-\frac{1}{n} \cos a\right)}
$$

et que la rupture se produit par cisaillement le long d'un plan incliné de $\alpha$ par rapport à l'horizontale. Enfin l'on a

$$
\operatorname{tg} \alpha=\frac{1}{\sqrt{1+\frac{1}{n^{2}}}-\frac{1}{n}} .
$$

4 Pour un matériau purement cohérent c'est-à-dire où $1 / n=0$ et $\alpha=45^{\circ}$ on a

$$
Q=2 c A
$$

5 En appliquant les résultats 1) et 2) Coulomb montre que lorsqu'il est en équilibre limite, un remblai derrière un mur de soutènement vertical de hauteur $\mathrm{H}$. se rompt suivant un plan incliné sur l'horizontale de ce même angle $\alpha$, et que la poussée totale sur le mur $P_{a}$ est :

$$
\mathrm{P}_{\mathrm{a}}=\mathrm{mH}^{2}-\mathrm{c \ell H}
$$


où

$$
\mathrm{m}=\frac{\gamma}{2 \operatorname{tg}^{2} \alpha} \text { et } \ell=\frac{2}{\operatorname{tg} \alpha} .
$$

6 De plus la pression unitaire à la profondeur z est

$$
\mathrm{p}_{\mathrm{a}}=2 \mathrm{mz}-\mathrm{c} \ell \text {. }
$$

7 Et en intégrant le moment de la pression $p_{\mathrm{a}}, \mathrm{dz}$ par rapport à la base entre les limites $\mathrm{z}=0$ et $\mathrm{z}=\mathrm{H}$, le moment agissant sur le mur est :

$$
M=\frac{1}{3} \mathrm{mH}^{3}-\frac{1}{2} \mathrm{C} \ell \mathrm{H}^{2} \text {. }
$$

8 L'angle $\alpha$ n'est pas l'inclinaison du terrain naturel comme l'avaient admis tous les auteurs antérieurs et il est indépendant de la cohésion.

9 En posant $\mathrm{P}_{\mathrm{a}}=0$ dans l'équation (5) on trouve de suite la hauteur limite d'un talus vertical sans support

$$
H_{c}=\frac{c \ell}{m}=\frac{4 c}{\gamma} \operatorname{tg} \alpha \text {. }
$$

10 Dans l'analyse ci-dessus la surface supérieure du terrain ou du remblai est horizontale. Pour appliquer ce résultat numériquement Coulomb admet que le talus naturel d'une masse de terre fraîchement déversée est de $45^{\circ}$ (i.e. $\dot{n}=1$ si $c=0$ ) et s'inspirant de Bélidor majore l'épaisseur du mur de $25 \%$ comme facteur de sécurité. II arrive ainsi à la règle pratique pour la stabilité contre le renversement qu'un mur ayant une face inclinée de 1/6:1. l'épaisseur en crête devrait être de $H / 7$, c'est-à-dire que l'épaisseur moyenne (à la mi-hauteur) serait juste au-dessous de $0,25 \mathrm{H}$.

11 II constate, sans le calculer, l'existence d'une butée si le mur est appliqué au terrain. Elle doit être supérieure à $\frac{1}{2} \gamma \mathrm{H}$ de même que la poussée doit être inférieure à celle-cı.

12 Enfin Coulomb s'attaque à l'effet du frottement du mur et en tire les expressions de la poussée en fonction de l'angle du plan de glissement et de cet angle même (qui est plutôt inférieur à $\alpha$ en ne tenant pas compte du frottement du mur). Les expressions sont compliquées avec ses notations, mais si on substitue $\operatorname{tg} \Phi=\frac{1}{n}$ et si on prend $c=0$ pour simplifier. on obtient l'équation (9) telle qu'elle se trouve sur la figure 3 .
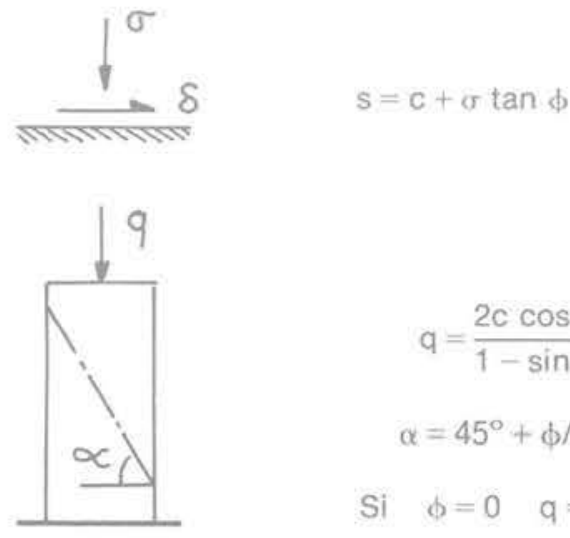

$$
\begin{gathered}
q=\frac{2 c \cos \phi}{1-\sin \phi}=\frac{2 c}{\tan \varepsilon} \\
\alpha=45^{\circ}+\phi / 2=90^{\circ}-\varepsilon \\
\text { Si } \phi=0 \quad q=2 c \quad \alpha=45^{\circ}
\end{gathered}
$$

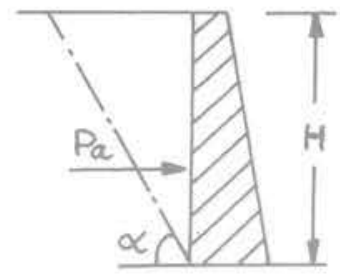

$$
\begin{aligned}
P_{\mathrm{a}} & =\frac{1}{2} \gamma \mathrm{H}^{2} \frac{1-\sin \phi}{1+\sin \phi}-2 \mathrm{cH} \frac{\cos \phi}{1+\sin \phi} \\
& =\frac{1}{2} \gamma \mathrm{H}^{2} \tan ^{2} \varepsilon-2 \mathrm{cH} \tan \varepsilon
\end{aligned}
$$

$$
\mathrm{p}_{\mathrm{a}}=\gamma z \tan ^{2} \varepsilon-2 \mathrm{c} \tan \varepsilon
$$

$$
\delta=0
$$

$$
M=\int_{0}^{H} P_{a}(H-z) d z
$$

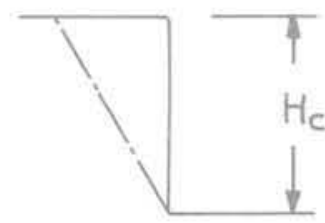

$$
H_{c}=\frac{4 c}{\gamma} \cdot \frac{\cos \phi}{1-\sin \phi}=\frac{4 c}{4 \tan \varepsilon}
$$

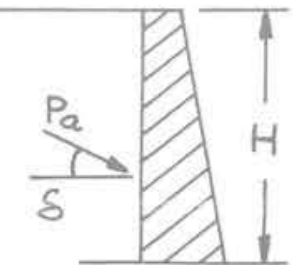

Si $\quad c=0$

$$
\mathrm{P}_{\mathrm{a}} \cos \delta=\frac{\gamma \mathrm{H}^{2}}{2} \cdot \frac{\cos ^{2} \phi}{\left[1+\sin \phi \sqrt{1+\frac{\tan \delta}{\tan \phi}}\right]^{2}}
$$


Dans un exemple numérique, en prenant $n=1$ et $\mathrm{c}=0$ et le frottement sur le mur égal au frottement interne, Coulomb trouve que la composante horizontale de la poussée $\left(\mathrm{P}_{\mathrm{a}} \cos \delta\right)$ est $0,125 \frac{\gamma \mathrm{H}^{2}}{2}$. Ceci d'après lui est une valeur trop basse, car le frottement du sol sur la maçonnerie est moindre que le frottement interne. II faut également tenir compte du fait que de l'eau peut s'infiltrer dans le remblai, réduisant le frottement interne et, même si l'on a prévu un drainage, exerçant une pression hydrostatique sur le mur. Ainsi il arrive à nouveau à la conclusion que dans la pratique les murs de soutènement devraient avoir une épaisseur moyenne égale au quart de leur hauteur.

Même aujourd'hui l'exposé de Coulomb n'est pas d'une lecture facile. A l'époque de sa publication, l'originalité de son raisonnement, les difficultés des notations et la conclusion, alors extraordinaire, que le plan de glissement était beaucoup plus raide que l'angle du terrain en place, a empêché que son importance fondamentale soit reconnue.

Le remplacement de $\operatorname{tg} \beta_{n}$ par $\frac{1}{n}$ a été fait par Reinhard Woltman 1753-1837 dans sa " contribution à l'architecture hydraulique $n$. Vol. 3 (Göttingen, 1794) et c'est lui qui a donné le premier l'expression qui nous est devenue familière $(\mathrm{si} \mathrm{c}=0$ )

$$
P_{a}=\frac{1}{2} \gamma H^{2} \frac{1-\sin \beta_{n}}{1+\sin \beta_{n}}
$$

Un autre pas vers l'acceptation de la théorie de Coulomb a été la publication des Recherches sur la Poussée des Pierres (Paris, 1802) par G.C. M. Riche de Prony (1755-1830), Directeur de l'École des Ponts et Chaussées et Professeur de Mécanique à l'École Polytechnique. II a introduit le paramètre $\eta$ où $\operatorname{tg} \eta$ égale le $n$ de Coulomb. On a alors $\eta=90-\Phi$ et, avec un remblai horizontal et un frottement nul du sol sur le mur, le plan de glissement est incliné de $\varepsilon=\frac{\eta}{2}$ par rapport à la face arrière du mur.

Si donc $\mathrm{c}=0$ le plan de glissement est la bissectrice de l'angle entre le mur et le talus naturel.

Dans la figure 3 les différentes solutions obtenues par Coulomb sont indiquées en termes de $\Phi$ et de $C$.

\section{Biographie}

- Charles Augustin Coulomb (1736-1806), a étudié sous la direction de Bossut à l'Ecole du Génie de Mézières, a servi dans le génie militaire de 1762 à 1781 à Brest, la Martinique, Bouchain, Cherbourg. Besançon et Rochefort, a ensuite été en poste à Paris et a pris sa retraite en 1791. Membre de l'Académie des Sciences, il acquit la notoriété par des recherches sur l'électricité et le magnétisme qu'il fit à la fin des années 1780 . Outre l'Essai de 1776, il a publié d'autres contributions à la science de l'ingénieur (Dictionnaire de Biographie Scientifique, 1791).

\section{MAYNIEL - 1808}

Traité expérimental, analytique et pratique de la poussée des terres et des murs de revêtement.

Paris - Colas - 1808
Divers essais de pression de terrain ont été exécutés au $18^{\circ}$ siècle et ont été décrits par Mayniel qui s'est efforcé de trouver ce qui avait pu être publié ou écrit à cet égard et de rendre compte des expériences qui avaient été faites. Mais les premiers essais vraiment significatifs sont ceux qu'il a faits en 1806 et 1807 avec l'appareillage de la figure 4 . La boite, de $3 \mathrm{~m}$ de long et $1,5 \mathrm{~m}$ de large, a, à une extrémité, un fond qui pivote autour d'une charnière située à sa base.

Avant de faire un essai, la caisse de bois $M$ est remplie d'eau et on la charge suffisamment pour résister à la pression latérale sur la paroi extrême lorsqu'on déverse du sable ou de la terre dans la boîte. On laisse alors s'échapper de l'eau de M jusqu'à ce que la paroi cède et qu'une surface de glissement se produise dans la masse. Une fois notés le mouvement de la paroi et l'emplacement du glissement on fixe la paroi d'extrémité et on retire le butoir $\mathrm{K}$. On mesure au moyen d'une corde, d'une poulie et d'un récipient gradué la force nécessaire pour obtenir le déplacement de $M$. Elle est égale à la composante horizontale de la pression de terrain quand le glissement s'est produit. Mayniel fit des essais sur de la terre meuble et de la terre compactée, sur un sol mélangé à du gravier et sur du sable meuble. La partie supérieure du remplissage était à la hauteur du sommet de la boîte ou comportait une surcharge; et la butée était placée à différents niveaux au-dessus de la charnière. Mayniel put conclure de ces essais que le point d'application de la pression était situé au tiers inférieur de la paroi pivotante, au moins pour les matériaux simplement déversés. Dans les essais sur un remplissage de terre le plan de glissement était incliné à environ $62^{\circ}$ sur l'horizontale et une poussée s'est développée après que la partie supérieure de la paroi mobile se soit déplacée d'environ $10 \mathrm{~cm}$.

Je me bornerai à analyser les essais sur la terre meuble et le sable, sans surcharge. Pour ces matériaux, le tableau 1 donne l'angle naturel de la pente, le poids unitaire, et la pression horizontale $(\mathrm{P} \cos \delta)$ par mètre de largeur, en même temps que les valeurs $\frac{1}{2} \gamma \mathrm{H}^{2}$ et $P \cos \delta / \frac{1}{2} \gamma H^{2}=K \cos \delta$.

Tableau 1

Résultats des essais de pression de terrain par Mayniel

\begin{tabular}{|c|c|c|}
\hline & terre & sable \\
\hline$\beta_{n}$ & $45^{\circ}$ & $37^{\circ}$ \\
$\gamma \mathrm{t} / \mathrm{m}^{3}$ & 1,10 & 1,36 \\
$\mathrm{P} \cos \delta \mathrm{kg}$ & 155 & 288 \\
$\frac{1}{2} \gamma \mathrm{H}^{2} \mathrm{~kg}$ & 1240 & 1530 \\
$\mathrm{~K} \cos \delta$ & 0,125 & 0,188 \\
\hline
\end{tabular}

Les valeurs de $K \cos \delta$ correspondent presque exactement avec la théorie de Coulomb, telle qu'elle résulte de l'équation (9) - fig, 3 , si $\beta_{n}=\Phi=\delta$. Mais dans la disposition des essais de Mayniel, (fig. 4), remarquable, la largeur de la caisse est trop faible par rapport à sa hauteur, pour éviter un frottement latéral appréciable. Si, par exemple, la poussée horizontale mesurée est, de ce fait, trop basse de $10 \%$, les valeurs corrigées de $\mathrm{K} \cos \delta$ correspondent exactement à la théorie de Coulomb avec $\delta=\frac{2}{3} \Phi$. 


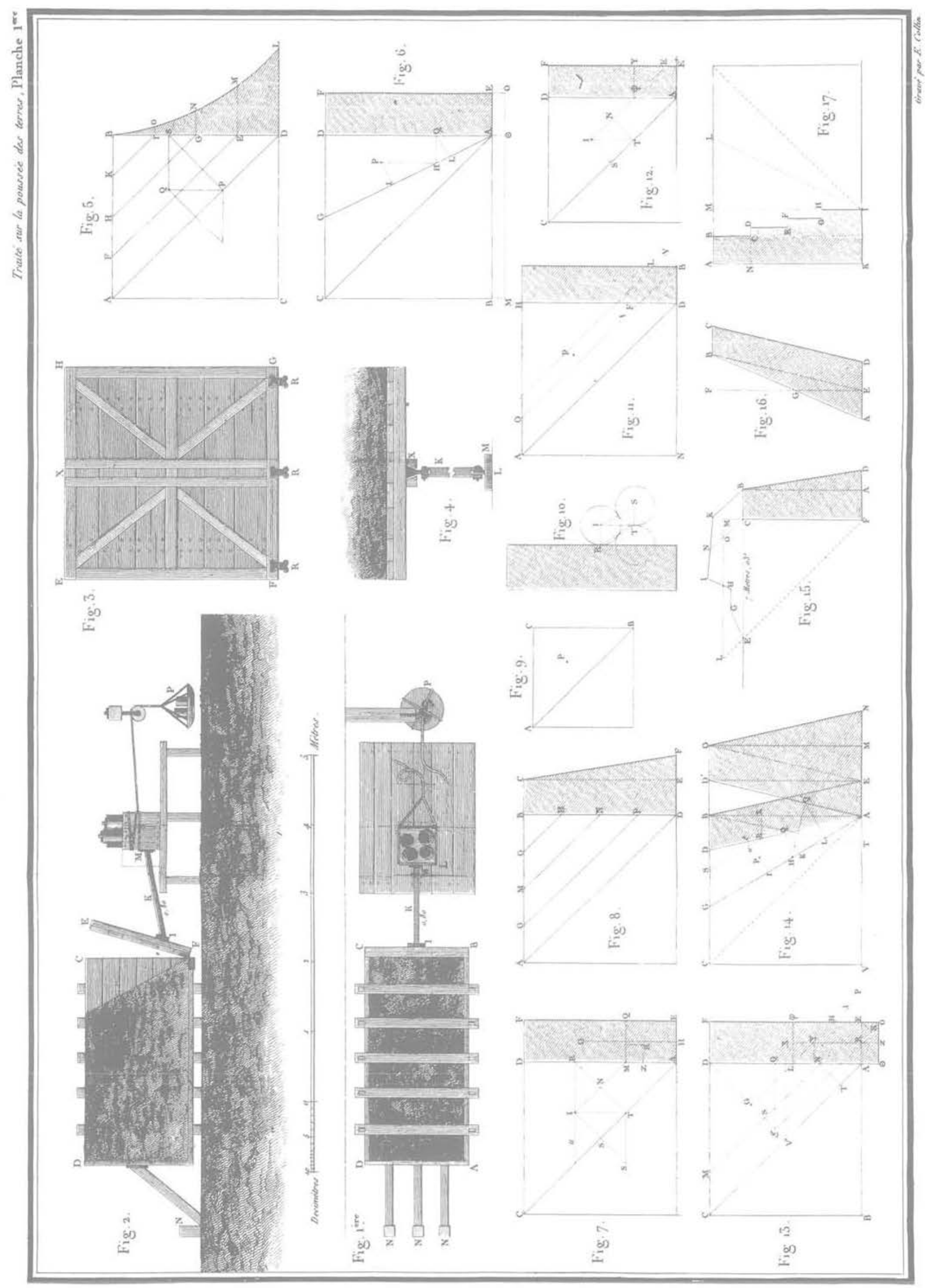

Fig. 4 
II faut noter toutefois que Mayniel, s'il indique comment on arrivait à l'équation (9) n'en a pas donné la solution.

De plus, par un processus qu'il m'a été impossibie de comprendre, il a tiré de ses essais des coefficients de frottement interne nettement inférieurs à $\operatorname{tg} \beta_{n}$. Cette erreur réduit malheureusement la valeur de sa contribution, mais les essais, eux-mêmes, ont une valeur importante.

\section{Biographie}

- Jean-Henri Mayniel (1760-1809) a commencé sa carrière dans les Ponts et Chaussées, puis en 1792 fut transféré dans le génie militaire. II devint Chef de Bataillon et mourut en Espagne pendant la guerre de Napoléon contre ce pays. (Renseignements communiqués par Monsieur Armand Mayer d'après les Archives du Génie.)

\section{FRANCAIS - 1820}

Recherches sur la poussée des terres, sur la forme et les dimensions des murs de revêtement, et sur les talus d'excavations. Mémorial de l'Officier du Génie N ${ }^{\circ} 4$ (1820) pp. 157-206.

Français commence son exposé en étendant l'analyse de Coulomb au calcul de la poussée sur des murs ayant une paroi arrière inclinée, avec un frottement nul sur le mur, et la surface du remblai horizontale. Sa solution est exacte.

II présente ensuite une discussion sur les projets de murs de soutènement, en y comprenant un coefficient de sécurité. Mais la partie la plus intéressante de son étude concerne la stabilité des pentes d'argile. II considère une entaille de profondeur $\mathrm{H}$ avec une tranchée inclinée de $\beta$ sur l'horizontale et il montre que l'équilibre limite d'une surface de glissement plane répond à l'expression

$$
H=H_{c} \frac{\sin \beta(1-\sin \Phi)}{1-\cos (\beta-\Phi)}
$$

où $\mathrm{H}_{\mathrm{c}}$ est l'expression de la hauteur verticale critique d'après Coulomb

$$
H_{c}=\frac{4 c}{\gamma} \frac{\cos \Phi}{1-\sin \Phi} .
$$

L'équation (1) peut donc être décrite sous la forme suivante :

$$
H=\frac{4 c}{\gamma} \frac{\sin \beta \cos \Phi}{1-\cos (\beta-\Phi)}
$$

et ceci est la première relation analytique relative à la stabilité des pentes d'argile, autres que pour $\beta=90^{\circ}$.

II est également intéressant de noter que Français reconnaît trois propriétés de base des sols; le poids unitaire $\gamma$, l'angle de talus naturel $\Phi$ (il utilise en fait $\eta=90-\Phi)$ et la hauteur critique $H_{c}$. L'angle de talus naturel est mesuré en déversant le sol à l'état meuble. lorsque $\mathrm{c}=0$. Pour déterminer la hauteur critique, il propose de faire des sections verticales de profondeurs différentes, de les laisser exposées pendant plusieurs mois aux conditions climatiques et de prendre pour $\mathrm{H}_{c}$ la plus grande hauteur qui a tenu au cours de cet essai. Français indique que ces trois paramètres devraient être dèterminés avec soin et que le résultat en serait d'une grande utilité.
Cette présentation a été adoptée par Navier (17851836) dans son célèbre Résumé des leçons données à l'École des Ponts et Chaussées sur l'Application de la Mécanique à l'établissement des Constructions (Paris 1833).

II donne quelques valeurs de c basées sur des hauteurs critiques comprises entre 1 et $4 \mathrm{~m}$. Mais il n'en déduit pas de conclusions intéressantes.

\section{Biographie}

- Jacques-Frédéric Français (1775-1833), ancien élève de l'Ecole Polytechnique, a servi dans l'Armée de 1801 à 1811. II devient alors professeur de fortifications et de géodésie à I'Ecole Militaire de Metz. (Renseignements communiqués par Monsieur Armand Mayer, d'après les Archives du Génie.)

\section{GARIDEL - 1840}

Note sur la Poussée et la Butée des Terres. Imprimé en appendice dans le traité de Poncelet $\left(N^{\circ} 9\right)$.

En 1836 Poncelet a discuté ses recherches sur la théorie des pressions de terrain avec Monsieur le Capitaine du Génie de Garidel, lequel, comme on l'a appris par la suite, était déjà arrivé à des résultats analogues par une analyse semblable à celle de Français. Poncelet apprécia la note que Garidel écrivit à cette époque au point qu'il l'introduisit mot pour mot dans son traité.

Le point principal est que Garidel donne une expression pour la butée. Comme Français, il suppose un mur incliné, admet un frottement sur le mur nul et suggère que la face supérieure du remblai est horizontale; enfin il utilise $\eta$ plutôt que $\Phi$. Son expression pour la poussée est la même que celle antérieurement obtenue par Français, mais sous une forme plus élégante. Pour un mur vertical la butée est donnée par l'expression

$$
P_{D}=\frac{1}{2} \gamma H^{2} \frac{1+\sin \Phi}{1-\sin \Phi}+2 \mathrm{CH} \frac{\cos \Phi}{1-\sin \Phi} .
$$

\section{Biographie}

- Mademoiselle Lacrocq, bibliothécaire de la Direction du Génie au Ministère de la Défense Nationale indique que le seul officier auquel ce travail pourrait être attribué est Bruno Charles François Garidel-Thoron (1807- $\quad$ ). Il passa par l'Ecole Polytechnique et l'Ecole du Génie de Metz, quitta l'Armée comme Capitaine du Génie en 1843 .

\section{PONCELET - 1840}

Mémoire sur la stabilité des revêtements et de leurs fondations.

Paris - Bachelier - 1840

II s'agit là d'un traité classique sur le calcul des murs de soutènement et leurs fondations, basé sur la théorie du coin de Coulomb et l'hypothèse $\mathrm{c}=0$. II contient 
beaucoup d'éléments nouveaux. Je ne donnerai ici qu'un résumé des principales contributions de l'auteur à la mécanique des sols.

1 Poncelet établit la valeur de la poussée et de la butée dans le cas général en tenant compte du frottement du mur et de l'inclinaison des deux parois de celui-ci. Il donne également une méthode graphique pour la détermination du plan de glissement critique et la poussée. Cette méthode a été très répandue et on peut la retrouver dans des traités modernes (voir Taylor (1948). Les expressions analytiques originales ont été réécrites par Müller-Breslau, Poussée sur les murs de soutènement (Stutgart, 1906) et c'est sous cette forme qu'elles sont présentées comme équations (1) et (2) pour un mur vertical dans la figure 5 . L'équation (9) de la fig. (3) correspond au cas particulier où $\beta=0$.

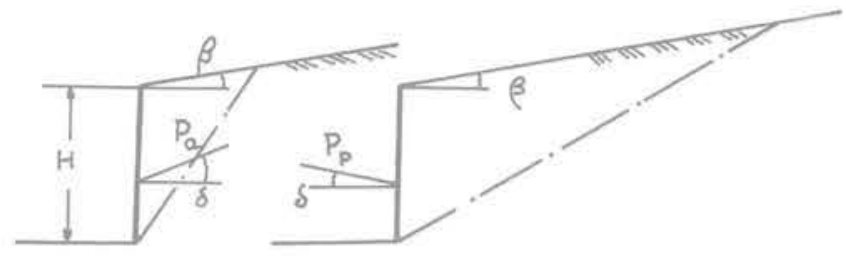

$P_{a} \cos \delta=\frac{\gamma H^{2}}{2} \frac{\cos ^{2} \phi}{\left[1 \pm \sqrt{\frac{\sin (\phi+\delta) \sin (\phi \mp \beta)}{\cos \delta \cos \beta}}\right]^{2}}$

\section{Fig. 5 D'après Poncelet, 1840}

2 Poncelet insiste sur l'importance de la stabilité des fondations au glissement et aux grands déplacements verticaux. II s'attaque pour la première fois à la théorie de ces problèmes. Au début de son analyse, il cite un rapport de Vauban, écrit en 1699 où ce grand ingénieur cite la rupture à Ypres de murs de 20 à 25 pieds de haut $(6$ à $8 \mathrm{~m})$ par glissement vers l'avant sur une surface de glissement dans une argile "savonneuse" qui, après la pluie, pouvait à peine se tenir sur une pente de $2 / 1$. Des accidents analogues se produisirent à Bergues en 1778 et Poncelet cite le glissement d'un mur à Soissons, aussi sur de l'argile, en 1837 (fig. 6).

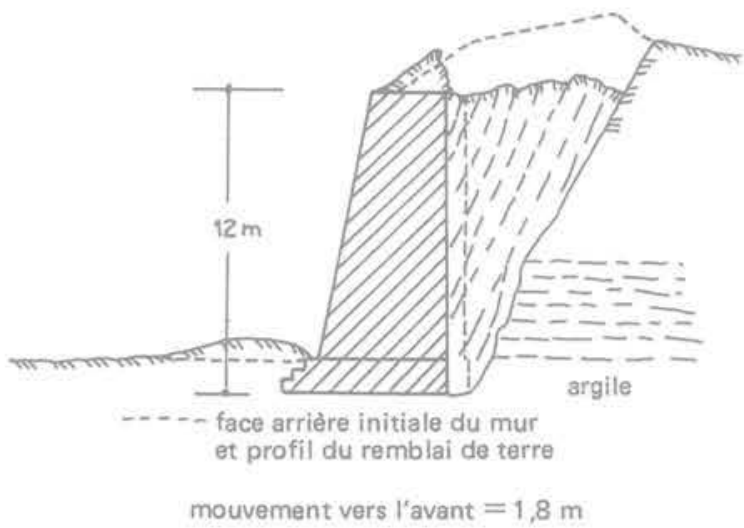

Fig. 6 Rupture d'un mur de soutènement à Soissons, 1837. Poncelet, 1840

II est pour cela absolument nécessaire, ou bien d'ancrer le mur assez profondément pour obtenir une butée suffisante sur la face aval de la fondation, et de baser le calcul, si le terrain est mauvais, par exemple sur $\Phi=26^{\circ}(\operatorname{cotg} 26=2)$ ou s'il faut une fondation sur pieux, d'incliner les pieux à un angle de, par exemple, $10^{\circ}$ sur la verticale.
3 La composante horizontale de la poussée au niveau de la fondation une fois compensée par la butée à la profondeur D il faut en outre se protéger contre une rupture par cisaillement du terrain sous la fondation causée par la composante verticale $Q$ de la résultante (fig. 7). Des ruptures de ce type peuvent être représentées par des coins de butée ou de poussée avec une interface verticale poussée à une profondeur $z$ sous la face frontale.

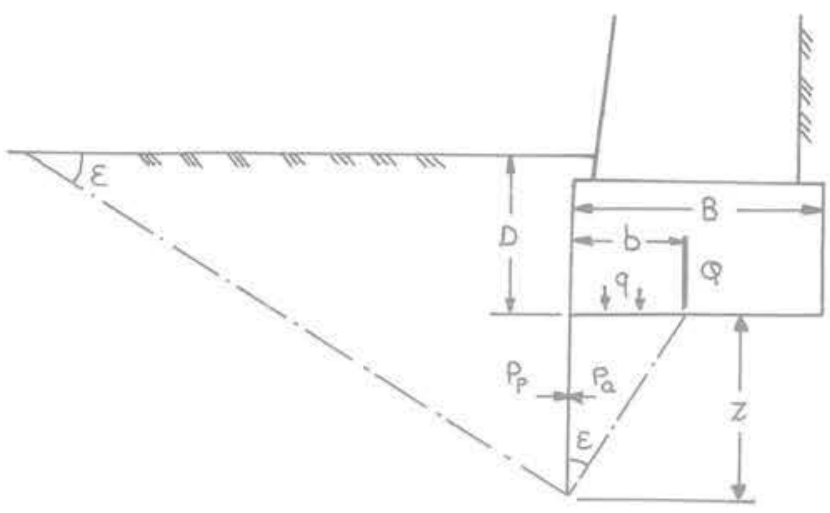

Équation de l'équilibre limite :

où

$$
q=\frac{\gamma D}{\tan ^{4} \varepsilon}+\frac{\gamma b}{2 \tan \varepsilon}\left[\frac{1}{\tan ^{4} \varepsilon}-1\right]
$$

$$
\varepsilon=(45-\phi / 2) \text {. }
$$

Fig. 7 Poncelet, 1840

Si l'on admet un frottement nul sur le mur, la butée que peut supporter cette interface est:

$$
P_{p}=\frac{\gamma}{2 \operatorname{tg}^{2} \varepsilon}\left[(D+z)^{2}-D^{2}\right]=\frac{\gamma}{\operatorname{tg}^{2} \varepsilon}\left[\frac{z^{2}}{2}+D z\right]
$$

où l'on a comme à l'ordinaire $\varepsilon=\frac{\pi}{4}-\frac{\Phi}{2}$.

De même, si q est la pression verticale (supposée uniforme) sur le coin de poussée, on a :

$$
P_{\mathrm{a}}=\gamma \operatorname{tg}^{2} \varepsilon\left[\frac{z^{2}}{2}+\frac{q z}{\gamma}\right]
$$

Mais pour l'équilibre limite, $\mathrm{P}_{\mathrm{a}}=\mathrm{P}_{\mathrm{p}}$ c'est-à-dire lorsque la pression sur la fondation provoque la rupture:

$$
q_{\mathrm{f}}=\frac{\gamma D}{\operatorname{tg}^{4} \varepsilon}+\frac{\gamma z}{2}\left[\frac{1}{\operatorname{tg}^{4} \varepsilon}-1\right] \text {. }
$$

Dans le cas d'une fondation bien étudiée sur un sol compressible la résultante $Q$ sera appliquée au milieu de la base $\mathrm{B}$ de manière à éviter le basculement. Dans ce cas $z=\frac{B}{2} \operatorname{tg} \varepsilon$. Mais d'une façon plus générale, si $Q$ agit à une distance b de l'angle de la face avant, on a

et

$$
\begin{gathered}
z=b \operatorname{tg} \varepsilon \\
q_{\mathrm{f}}=\frac{\gamma D}{\operatorname{tg}^{4} \varepsilon}+\frac{\gamma b}{2} \frac{1}{\operatorname{tg} \varepsilon}\left(\frac{1}{\operatorname{tg}^{4} \varepsilon}-1\right)
\end{gathered}
$$

où, si la fondation est symétriquement chargée

$$
\mathrm{b}=\frac{\mathrm{B}}{2} \text {. }
$$

Revenant à l'équation (3) il apparait que D atteint sa valeur maximale si $z=0$ lorsque :

$$
\mathrm{D}=\frac{\mathrm{q}}{\gamma} \operatorname{tg}^{4} \varepsilon .
$$


Ceci implique la rupture d'un élément du sol sous la face avant. Le même résultat peut également être obtenu de suite en considérant l'équilibre des contraintes sur cet élément. Mais l'équation (5) correspond à une méthode prudente de calcul de $\mathrm{D}$.

De même il est prudent de vérifier que $B$, ou plus généralement $b$, satisfait à l'équation (4) lorsque $D=0$. Si ces deux règles sont appliquées, le tassement de la fondation sera uniquement celui dû à la compressibilité du sol, tout danger de rupture par cisaillement ayant été évité.

Dans le langage technologique moderne on voit que dans l'équation (4) Poncelet exprimait que pour une fondation chargée en son centre on avait :

$$
\begin{array}{r}
N_{a}=\frac{1}{\operatorname{tg}^{4} \varepsilon} \\
N \gamma=\frac{1}{2 \operatorname{tg} \varepsilon}\left[\frac{1}{\operatorname{tg}^{4} \varepsilon}-1\right] .
\end{array}
$$

Lorsque $\Phi$ est compris entre 25 et $35^{\circ}$ ce résultat est légèrement supérieur aux valeurs de ces coefficients donnés par Terzaghi pour des ruptures locales par cisaillement.

4 Poncelet fait la remarque intéressante confirmée par l'expérience, que les contreforts réduisent la pression de terrain par effet de voute.

5 Enfin, on peut noter qu'il utilise peut être le symbole $\Phi$ pour l'angle de frottement interne du sol pour la première fois dans la littérature française; s'il prend $\mathrm{c}=0$ dans l'argile c'est qu'il admet que la cohésion s'annule à l'instant qui précède la rupture et qu'au moment où la rupture a effectivement lieu, la résistance au cisaillement est $\sigma \operatorname{tg} \Phi$.

\section{Biographie}

Jean Victor Poncelet (1788-1867), après avoir servi dans le Génie, devint professeur de mécanique à Metz en 1824 et à la Sorbonne en 1837; membre de l'Académie des Sciences on lui doit des travaux originaux en géométrie pure, mécanique appliquée et hydraulique. [Dictionnaire de Biographie Scientifique (1975).]

\section{HOPE - 1845}

Compte-rendu d'Essais exécutés à Chatham par feu le Lieutenant Hope, du Royal Génie, sur la pression de la terre sur les soutènements et la meilleure forme de ceux-ci. Rapports sur des sujets relatifs aux missions du Corps du Royal Génie - Vol. 7 (1845) pp. 69-86.

Le Lieutenant Hope, alors jeune officier à l'état-major du Royal Engineering, a exécuté en 1842-43 des essais sur les pressions de terrain sur la résistance comparée de modèles de murs de soutènement à grande échelle. II mourut avant de pouvoir rédiger les résultats auxquels il était arrivé, mais en hommage à son travail on publia ce rapport basé sur ses résultats et ses notes.

Ses essais ont été faits sur du sable sec ayant un angle d'inclinaison naturel de $35^{\circ}$ et un poids spécifique de 91 livres/pied cube à l'état meuble $\left(14,4 \mathrm{kN} / \mathrm{m}^{3}\right)$, dans une boite en bois de 2 pieds de long $(0,60 \mathrm{~m})$ et 1 pied carré $\left(0,09 \mathrm{~m}^{2}\right)$ de section. Comme Mayniel, dont il avait lu le livre, Hope réduisit la poussée horizontale appliquée au « mur n formant l'extrémité de la boîte, jusqu'à obtenir la rupture. Mais avec une technique expérimentale très améliorée, il mesura simultanément les composantes horizontale et verticale de cette pression. Ceci lui permit de déterminer le frottement sur le mur, $\operatorname{tg} \delta$. On trouvera dans le tableau 2 la moyenne de deux essais avec un mur lisse et de 7 essais avec un mur rugueux pour $\mathrm{K} \cos \delta=$

$$
\mathrm{P} \cos \delta / \frac{1}{2} \gamma \mathrm{H}^{2} \text {. }
$$

Tableau 2

Résultats d'essais de pression de terrain par le Lt Hope

\begin{tabular}{|l|c|c|}
\hline & mur lisse & mur rugueux \\
\hline $\mathrm{P} \cos \delta$ (en livres) & 10,0 & 9,4 \\
$\delta$ & $c .8^{\circ}$ & $27^{\circ}$ \\
$\frac{1}{2} \gamma \mathrm{H}^{2}$ (en livres) & 45,5 & 45,5 \\
$\mathrm{~K} \cos \delta$ & 0,220 & 0,207 \\
\hline
\end{tabular}

Les valeurs de $\mathrm{K} \cos \delta$ mesurées étaient environ $10 \%$ inférieures à celles calculées par l'équation (9) (fig. 3). Cette différence peut être attribuée en partie à l'effet du frottement latéral dans la boîte et en partie au fait que l'angle de frottement interne $\Phi$ peut être légèrement supérieur à l'angle de talus naturel $\beta_{n}$.

Des essais complémentaires furent exécutés avec une surcharge inclinée à $\beta_{n}$. Mais ces essais sont peu représentatifs en raison de la variation rapide de $K \cos \delta$ lorsque $\beta$ tend vers $\Phi$.

Hope fit aussi des essais avec des couches de sable de différentes couleurs et avec une boîte comportant une paroi de verre. Ces essais montrèrent la ligne de rupture lorsque le mur ètait déplacé vers l'avant. Cette ligne était parfois toute droite, mais plus souvent légèrement concave. Dans ces essais, avec différentes inclinaisons du mur, la surface de glissement fut trouvée plutôt plus inclinée que la théorie de Coulomb ne l'aurait indiqué.

Dans ses essais sur modèle réduit Hope utilisa un mur à section rectangulaire, un autre avec des contreforts et un troisième également à contreforts mais incliné sur deux faces de 1/5:1. Il utilisait dans chaque cas le même nombre de briques par couche. Les murs étaient montés, un remplissage en terre étant placé en arrière du mur à mesure qu'une couche de briques était ajoutée jusqu'à rupture. Le mur vertical rectangulaire, large de 1 pied 11 pouces $(0.58 \mathrm{~m})$, se rompit lorsque sa hauteur atteignit 10 pieds $(3 \mathrm{~m})$. Le mur à contreforts tint jusqu'à 13 pieds $(4 \mathrm{~m})$ et le mur à parois inclinées jusqu'à 21 pieds $(6 \mathrm{~m})$. Ce dernier résultat confirme, nous le voyons, l'économie que le fait d'incliner les faces des murs apporta dans les grands travaux de Jessop et Rennie dans les docks de Londres plus de 40 ans auparavant.

\section{Biographie}

- Charles William Hope (1824-1844), plus jeune fils de l'éminent général Sir John Hope, fut nommé Officier du Royal Génie en juin 1842 et mourut dans la maison de sa mère à Edinburgh en mars 1844 à l'âge de 19 ans.

[Répertoire l'Armée Gentleman's Mag. Nouvelle série 21 - (1844) 557.] 
De grands progrès ont été faits au $19^{\mathrm{e}}$ siècle en mécanique des sols appliquée et études géotechniques. Je limiterai mon exposé à trois sujets :

1 La stabilité des pentes en terrains argileux, comprenant l'observation des surfaces de glissement et l'étude des remèdes, dont le drainage.

2 La constatation que les tassements de longue durée des fondations et des remblais assis sur de l'argile sont dus à la consolidation de celle-ci.

3 La construction de barrages sur fondations perméables.

Mais si le temps le permettait beaucoup d'autres questions pourraient être soulevées. Je citerai simplement :

a la consolidation par injection de rocher et de matériaux perméables,

b l'emploi de l'air comprimé dans le forage de tunnels et les fondations de caissons,

c les rabattements de nappes,

d l'évolution des formules de battage de pieux,

e la mesure du frottement latéral sur les pieux et les fondations circulaires,

f les progrès dans la technique des barrages en terre.

Que des progrès aient été faits sur ces différents points ressort des types de travaux de génie civil réalisés: voies ferrées, barrages, tunnels, ports et ponts construits à une échelle constamment croissante et, somme toute, avec un succès acceptable. Evidemment. il y a eu des échecs aussi bien que des succès, dus parfois à une conception fausse du génie civil, mais dans d'autres cas à une connaissance encore tout à fait insuffisante des propriétés du sol. Ceci nous paraît évident, mais il fallait le génie de Terzaghi pour résoudre ces problèmes et, plus que tout le reste, son effort de recherche des propriétés des sols et son insistance à déterminer la relation entre la théorie et la pratique qui a conduit à la mise au point de la mécanique des sols moderne.

\section{JESSOP et TELFORD - 1810; TELFORD - 1821}

a Rapport de William Jessop et Thomas Telford en date du 18 octobre 1809, dans le $7^{\mathrm{e}}$ Rapport de la Commission du Canal Calédonien pp. 22-24. (Compte-rendus parlementaires, 1810.)

b Thomas Telford, «la Navigation Intérieure », Encyclopédie d'Edinburgh, vol. 15 (1821), pp. 209-315.

L'écluse marine de Clachnaharry constituant l'entrée côté Est du Canal Calédonien a été construite de 1808 à 1812 sous la direction de Telford avec Jessop comme Ingénieur-Conseil. Cette écluse (de 180 pieds de porte à porte, $(55 \mathrm{~m}) 40$ pieds de largeur $(12 \mathrm{~m}) 25$ pieds de hauteur $(7,5 \mathrm{~m})$ d'eau sur le seuil externe par les marées de printemps), est située à 400 yards hors de la ligne des hautes eaux ( $366 \mathrm{~m}$ ). Les essais ont montré la présence de 55 pieds d'argile molle limoneuse $(16,5 \mathrm{~m})$ sous le niveau des fondations (fig. 8). Pour éviter d'avoir à construire un batardeau de grande dimension dans des conditions difficiles, et aussi pour améliorer l'argile molle, le site a été préchargé par un remblai d'argile à blocaux et des déchets de carrière à l'intérieur duquel l'emplacement de l'écluse fut excavé après six mois.
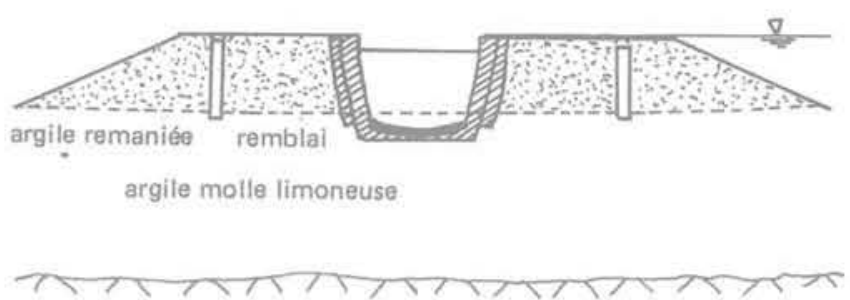

Fig. 8 Croquis du profil de l'écluse marine de Clachnaharry, 1808-1812. Canal Calédonien

Dans leur premier rapport à ce sujet (du 14 novembre 1808 , dans le $6^{\circ}$ rapport de la Commission, imprimé de 1809), Jessop et Telford indiquent que le remblai * aurait pour objet de comprimer ou d'exprimer l'argile molle de manière à obtenir une fondation résistante w. IIs font là une comparaison avec l'expression d'une éponge. Ceci devient clair lorsque nous lisons dans leur rapport suivant, du 18 octobre 1809, qu'ils ont décidé de monter le remblai temporairement audessus de la hauteur définitive "de manière, grâce à cette surcharge, de consolider l'argile molle plus rapidement et plus efficacement ". Et dans son article de 1821 Telford écrit que cette surcharge a eu pour objet d'exprimer l'eau et de consolider l'argile en lui appliquant une charge supérieure à celle de la maçonnerie de l'écluse.

On voit que dès 1809 Jessop et Telford avaient compris d'une façon générale le processus de la consolidation de l'argile et le principe du préchargement. L'explication de Telford, de 1821, bien que courte est parfaitement explicite. L'exécution de cette écluse qui a été terminée de façon satisfaisante peut être résumée comme suit :

remblai mis en place de novembre 1808 à décembre 1809

tassement pratiquement arrêté en mai 1810 ,

excavation juin 1810 -juin 1811 .

construction de la maçonnerie juin 1811 -août 1812.

\section{Biographies}

William Jessop (1745-1814) a été formé par le grand Ingénieur du $18^{\circ}$ siècle John Smeaton. II a eu la responsabilité du projet et de l'exécution de beaucoup de travaux importants parmi lesquels le Canal de Rochdale, le Grand Canal de Junction, les Docks de la Société des Indes Occidentales à Londres, et le pont flottant de Bristol (Charles Hadfield et A. W. Skempton: William Jessop, Ingénieur - Newton Abbot, 1979.)

Thomas Telford (1757-1834), F.R.S. et premier Président de l'Institution des Ingénieurs Civils Britanniques. Pendant une carrière longue et variée, il est apparu très éminent comme ingénieur de ponts, de routes, de canaux et de ports. (Sir Alexander Gibb : Histoire de Telford Londres, 1935.) 
Traité de construction routière où sont présentés les principes suivant lesquels les routes doivent être construites, comprenant les plans, spécifications et contrats utilisés par Thomas Telford dans la construction de route de Holyhead. Londres: Longman, 1833.

Au cours d'une longue expérience comme chargé de la route Londres-Holyhead, Parnell acquit une grande connaissance de la construction routière. Les tables relatives aux pentes des déblais présentant toute sécurité dans les différentes couches (voir tableau 3) offrent un certain intérêt.

\section{Biographie}

Sir Henry Brooke Parnell (1776-1842), Membre du Parlement, Economiste et Membre d'Honneur de I'Institution des Ingénieurs Civils Britanniques; a été avant tout responsable de la constitution de la Commision de la Route de Holyhead. (Dictionnaire de Bibliographies Nationales, 1895 - Vie de Thomas Telford, Londres, 1838.)

Tableau 3

Sécurité des pentes des tranchées par Sir Henry Parnell, 1833

\begin{tabular}{l|c|c|}
\hline Argile de Londres & $3 / 1$ & $18,5^{\circ}$ \\
Argile d'Oxford & $2 / 1$ & $26,5^{\circ}$ \\
Argile d'Oxford & & \\
en tranchées profondes & $3 / 1$ & $18,5^{\circ}$ \\
Craie & $1 / 1$ & $45^{\circ}$ \\
Calcaire ou grès & $0,25 / 1$ & $76^{\circ}$ \\
$\begin{array}{l}\text { Calcaire ou grès avec } \\
\text { intercalations d'argile : } \\
\text { lits horizontaux }\end{array}$ & $1,5 / 1$ & $33,5^{\circ}$ \\
$\begin{array}{l}\text { Si les couches sont inclinées, } \\
\text { les pentes peuvent devoir }\end{array}$ & & \\
être inclinées à & $4 / 1$ & $14^{\circ}$ \\
\hline
\end{tabular}

\section{GREGORY - 1844}

Déblais et remblais de voies ferrées avec un compte-rendu de quelques glissements dans l'argile de Londres sur la ligne de Londres à Croydon.

Min. Proc. Inst. Civil Eng., vol. 3 (1844), pp. 135-145.

Premier compte-rendu d'un glissement dans l'argile de Londres. II se produisit brutalement le 2 novembre 1841 près de la gare de New Cross, trois ans après l'exécution du déblai. En 4 heures environ, 50000 yards cubes d'argile $\left(36000 \mathrm{~m}^{3}\right)$ se déplacèrent sur une surface de glissement d'apparence "vitrifiée " le long du contact entre l'argile brune et bleue (fig. 9). Le travail de déblaiement était encore en cours le 26 novembre lorsqu'un glissement analogue se produisit sur la face opposée.

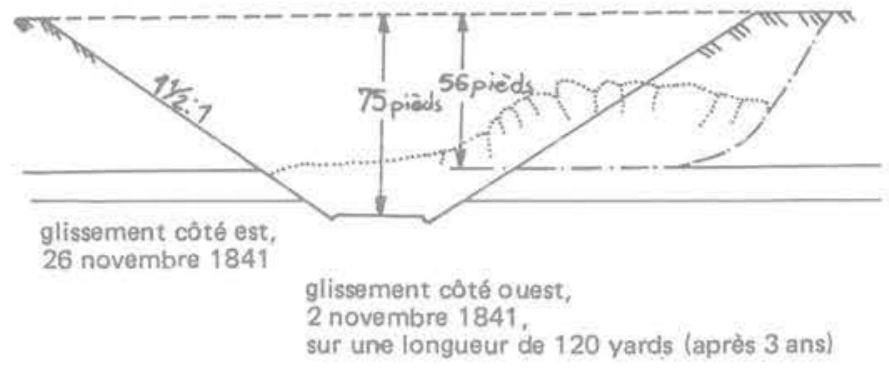

Fig. 9 Chemin de fer de Londres à Croydon. Tranchée exécutée en 1838. Coupe rectifiée d'après Gregory, 1844

Les déblais avaient été initialement découpés à la pente extraordinairement raide de 1,5:1 malgré la nature du sol et la grande profondeur (max 75 pieds) (23 m) de l'entaille. Les mesures prises pour remédier à la situation ont comporté la réduction de la pente à 2:1 avec de larges bermes d'oủ résultait une inclinaison moyenne de 2,5:1. Mais d'autres glissements, sur la même ligne, au sujet desquels il n'est pas donné de détails, ont été traités au moyen de tranchées remplies de gravier (ou contreforts) découpées au-dessous de la surface de glissement suivant les lignes de plus forte pente et réunies à la base par une couche de gravier.

De la longue et intéressante discussion sur le rapport il résulte que Robert Stephenson avait utilisé des contreforts pour stabiliser des glissements sur la ligne de Londres à Birmingham en 1839, notamment dans le Lias dans la tranchée de Blisworth près de Northampton. II y avait un accord général sur le fait que les contreforts constituaient des drains profonds et provoquaient une augmentation de la résistance, comme des butons de gravier ou de pierraille fondés sous la surface de glissement.

Dans la discussion, comme dans le rapport lui-même, il est fait allusion à des " joints et des fissures naturelles" dans l'argile de Londres (Gregory) et au fait qu'elle présente une grande quantité de fissures dans toutes les directions (de La Beche).

\section{Biographies}

Charles (devenu Sir Charles) Hutton Gregory (1817-1898) étudia avec Robert Stephenson et James Walker; fut Ingénieur délégué par William Cubitt sur les chantiers du Chemin de fer de Londres à Croydon; a succédé à Brunel en 1846 comme Ingénieur du Chemin de fer de Bristol à Exeter, et devint une autorité internationale comme Ingénieur des Chemins de fer: fut Président de I'Institution des Ingénieurs Civils [Min. Proc. Inst. Civ. Eng., 132 (1898) 377-382]. Sir Henry Thomas de La Beche (1796-1855), F.R.S., fut le premier Directeur du Service Géologique à partir de 1835. (Dictionnaire des Biographies Scientifiques, 1971.) 
14 COLTHURST - 1844

\section{Discussion du rapport de C. H. Gregory $\left(n^{\circ} 13\right)$}

Min. Proc. Inst. Civ. Eng., vol. 3 (1844), pp. 163-168.

Colthurst donne la première description qui ait été publiée d'un accident de talus dû à la fondation. $\mathrm{Ce}$ talus, sur le Great Western Railway, près de Hanwell, était haut de 54 pieds $(16 \mathrm{~m})$ avec des pentes inclinées à $1,5: 1$. II était assis sur 4 pieds d'argile alluvionnaire et 3 à 10 pieds ( 1 à $3 \mathrm{~m}$ ) de gravier superposés à l'argile de Londres (fig. 10). Le glissement, qui se produisit en mai 1837 , a causé un tassement de 30 pieds $(9 \mathrm{~m})$ suivi par un soulèvement de 10 pieds $(3 \mathrm{~m})$ sur une largeur d'environ 80 pieds $(24 \mathrm{~m})$ et une longueur de 400 pieds $(122 \mathrm{~m})$. Des désordres de moindre importance se prolongeaient jusqu'à 220 pieds du remblai $(67 \mathrm{~m})$.

Immédiatement après le glissement, I. K. Brunel, Ingénieur en Chef du G.W. R., fit construire une berme ou "terrasse " ainsi que des tranchées normales au talus jusqu'au pied de celui-ci. Celles-ci permirent de se rendre compte de la nature du terrain qui avait été

Fig. 10 a
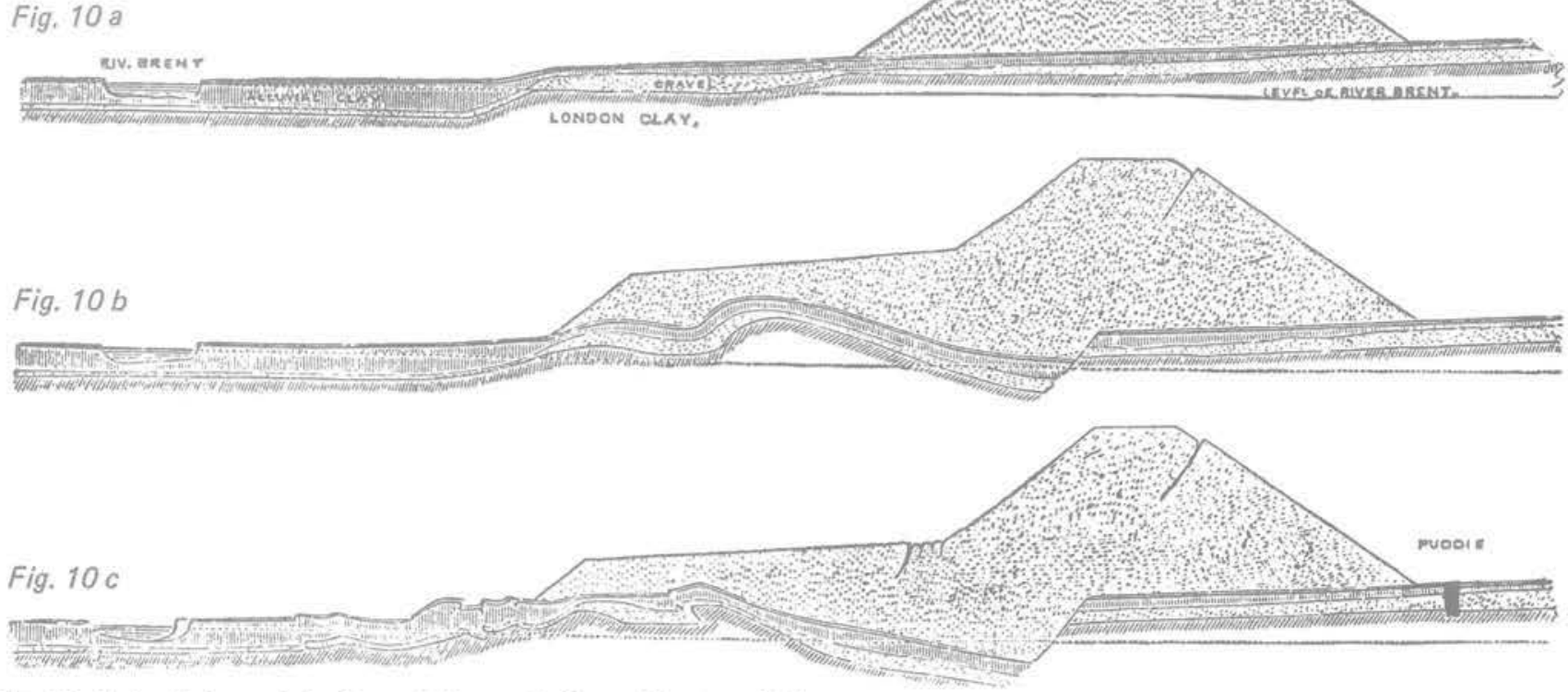

Fig. 10 Talus le long de la Brent à Hanwell, Great Western Railway

3 à 10 pieds $(1$ à $3 \mathrm{~m})$ de terrains sédimentaires assis sur de l'argile de Londres recoupée en toutes directions par des couches ou joints plastiques. Le terrain était en pente douce vers la rivière Brent qui se trouvait à 20 pieds environ au-dessous de la partie Sud du remblai $(6 \mathrm{~m})$

Le tassement du remblai a commencé durant la nuit du 21 mai 1837. Au matin on constata que la fondation avait cédé et qu'une masse de terrain, de 30 pieds de long $(9 \mathrm{~m})$ par 15 de large $(4,5 \mathrm{~m})$ avait été repoussée de sous la partie basse (Nord) du remblai vers le Brent. Pendant quatre mois cette avancée se développa et le tassement du remblai se poursuivit jusqu'à ce que la surface se présentât comme une ondulation. Une section à travers la partie déplacée montra que les différentes couches suivaient les déplacements de la surface. Des sections normales au remblai sur toute la hauteur montrée figures $10 \mathrm{~b}$ et $10 \mathrm{c}$ permirent de se rendre compte du déplacement des différentes couches. L'accident survenu au remblai à cette période était limité à un tassement d'environ 15 pieds avec une fissure tout le long de la partie supérieure de la pente Sud au côté opposé à celui où la fondation avait cédé. perturbé. Les tranchées furent très probablement remblayées en gravier pour servir de drains. On ne tint pas compte du terrain sous le remblai dans l'appréciation du tassement à la partie supérieure du glissement. La terrasse latérale se comporta comme un procédé de stabilisation efficace.

\section{Biographies}

Joseph Colthurst (1812-1882) Ingénieur, fut chargé de la section Hamwell-Iver du G.W.R. et ensuite de la section de voie jusque Didcot. II exécuta ultérieurement différents travaux sur des voies ferrées en Angleterre et à l'étranger. Min. Proc. Inst. Civ. Eng., 73 (1883), pp. 356-358.

Isambard Kingdom Brunel (1806-1859) le plus fameux ingénieur de voies ferrées de l'ère Victorienne; F. R. S. et Vice-Président de I'Institution des Ingénieurs Civils (L.T.C. Rolt: I. K. Brunel - Londres, 1957).

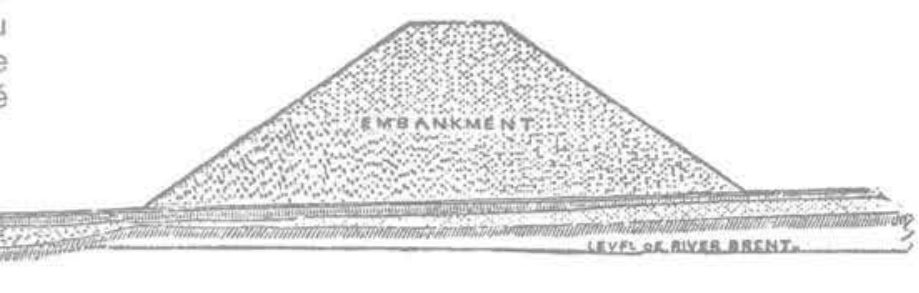


15 COLLIN - 1846

Recherches expérimentales sur les glissements spontanés de terrains argileux.

Paris : Carilian-Goeury et Dalmont, 1846.

Collin a fait une remarquable contribution à la connaissance de la stabilité des pentes argileuses, notamment quant aux premiers glissements de tranchées, de talus et de barrages en terre. Résumés autant que possible, les principaux points étudiés sont :

1 Les ruptures se produisent en principe le long de surfaces de glissement courbes (surface de glissement) de section approximativement cycioïdales, savonneuses, polies et striées et s'étendant à peu près jusqu'au pied de la pente. Un glissement est souvent précédé par des fissures en crête. Quelquefois, un second ou un troisième mouvement se produisent peu de temps après le premier donnant lieu à des glissements multiples (rétrogrades).

2 Les glissements profonds, tels qu'ils sont décrits ci-dessus, se produisent lorsque la cohésion est juste inférieure aux forces produites par la gravité. Ils peuvent se produire pendant ou immédiatement après la construction, ou ils peuvent être retardés de quelques années tandis que les infiltrations réduisent la cohésion.

3 Les mouvements suivant le premier glissement se poursuivent jusqu'à ce qu'une nouvelle position d'équilibre soit atteinte. La pente prend alors un profil définitif en $\mathrm{S}$. Ces mouvements sont alors contrariés par les frottements, la cohésion ayant été annulée. La résistance due au frottement peut être réduite par l'effet de la pluie pénétrant la masse d'argile perturbée.

4 En général une surface de glissement est le résultat et non la cause d'une rupture. Collin reconnaît la possibilité de surfaces de glissement préexistantes, mais il rejette cette explication, donnée par Girard, des glissements dans le Bois de Saint-Denis (Mémoires sur le Canal de l'Ourcq - Paris, 1831).

5 II y a aussi des glissements superficiels, peu profonds, dus à la destruction de la cohésion ou à sa réduction du fait de pluies répétées, de sécheresse, de gel et de dégel.

6 Collin décrit en détail, en donnant les profils cotés, dix glissements profonds. II se reporte également, avec croquis à l'appui, à un nombre important d'autres glissements. La surface de glissement, lors de l'accident survenu à la tranchée de la fondation du barrage de Gros-Bois (fig. 11). C'est probablement la première qui ait fait l'objet de mesures exactes; la première qui ait fait l'objet d'une publication (par C. J. Minard - Cours de Construction des Ouvrages qui établissent la navigation des rivières et des canaux - Paris, 1841) est le levé du troisième glissement (1836) du barrage de Cercey.

7 Collin apporte une grande attention aux remèdes, y compris le drainage et le recompactage, mais il insiste tout spécialement sur les contreforts en pierre cassée. Ceux-ci, à 9 ou $10 \mathrm{~m}$ l'un de l'autre, de $2 \mathrm{~m}$ à $2,5 \mathrm{~m}$ de large, sont entaillés sous la surface de glissement et agissent avant tout comme des butées internes. Ils ont été utilisés pour réparer le glissement de 1835 du barrage de Cercey (il semble que cela ait été la première utilisation de ce procédé en France) et ensuite sur la plupart des glissements survenus lors des travaux du Canal de Bourgogne. Dans le cas de glissements superficiels, le battage de pieux, les

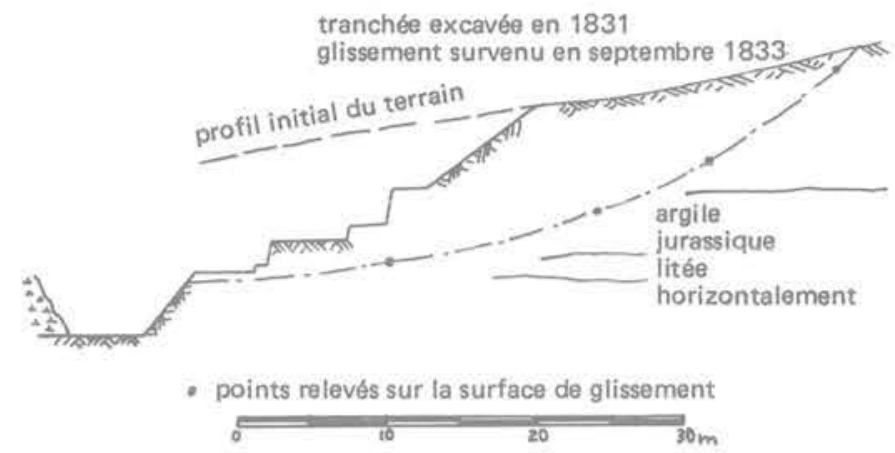

Fig. 11 Glissement dans la tranchée de la fondation, Grosbois. Collin, 1846

matelas de fascines, et les drains de faible profondeur peuvent être efficaces, ainsi que la plantation d'arbres.

8 Des essais de mesure de la cohésion ont été effectués dans lappareil de cisaillement représenté (fig. 12), sur des échantillons de section $16 \mathrm{~cm}^{2}$ ou de $1 \mathrm{~cm}^{2}$ dans le cas d'argiles très dures. Les résultats (en tonnes/mètre carré) peuvent être résumés comme suit :

Argile molle, telle qu'elle résulte

de l'infiltration d'eau

de source ou de pluie

$1,8-2,2$

Argile à la consistance du matériau

utilisé pour la construction

de remblais ou barrages

$3,2-5,9$

Argile compacte telle que celle

rencontrée dans les couches vierges

de l'époque secondaire ou tertiaire

$40-60$

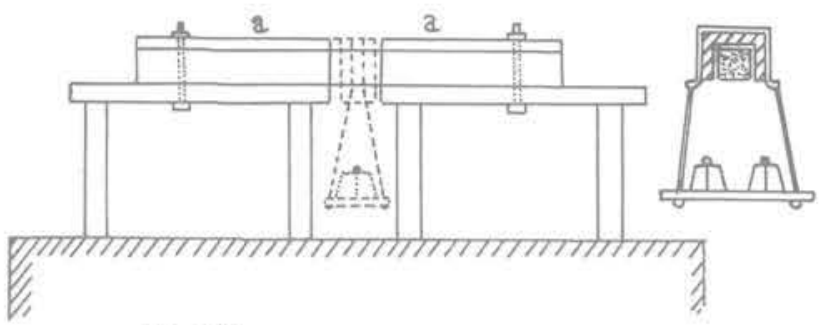

Fig. 12

Ces chiffres montrent l'influence de la teneur en eau sur la résistance au cisaillement. Collin a constaté que les essais en pratique avaient été trop rapides et il essaie de mesurer la résistance "permanente" en déterminant la résistance au cisaillement maxima que les échantillons puissent supporter sans déformation apparente. Elle semble être de l'ordre de $25 \%$ de la résistance "instantanée»,

9 Des essais de frottement ont été effectués par Collin en faisant glisser un bloc d'argile sur un autre. II trouva des coefficients de 0,6 à 1 , mais en humidifiant les surfaces, il trouve des coefficients d'environ 0,2 $\left(\Phi=12^{\circ}\right)$.

10 En raison des effets de l'eau et de la durée, il est difficile de fixer une valeur limite de la cohésion d'une argile qui corresponde à une stabilité permanente. Mais si l'on pouvait donner une telle valeur, une analyse simple montrerait si une pente est stable ou quelle résistance devraient comporter des contreforts pour assurer sa stabilité. Cette résistance $Q$ exprimée en tonnes par mètre de large peut être tirée approximativement de l'équation : 


$$
\mathrm{Q}=\mathrm{W} \sin \alpha-\mathrm{cL}
$$

où W est le poids des terres au-dessus d'une surface de glissement cycloïdale potentielle, $\alpha$ est l'inclinaison de la surface en un point à la verticale du centre de gravité de ces terres, L est la longueur de la surface de glissement et où $\mathrm{Q}$ est dirigé à l'angle $\alpha$. Pour l'équilibre limite on a les conditions :

$$
\begin{aligned}
Q & =0 \\
W \sin \alpha & =c L .
\end{aligned}
$$

A titre d'exemple numérique, pour une pente de 1,5:1 de $10 \mathrm{~m}$ de hauteur, avec $\gamma=1,725 \mathrm{t} / \mathrm{m}^{3}$, la valeur de $c$ est $1,7 \mathrm{t} / \mathrm{m}^{2}$ (valeur que I'on peut comparer avec celle de $c=2,4$ obtenue par l'analyse de la section supposée circulaire de Taylor).

De même, après le glissement on $\mathrm{a}$ :

$$
Q=W \sin \alpha-W \cos \alpha \times f
$$

où $f$ est le coefficient de frottement.

L'équation $\mathrm{W} \sin \alpha=\mathrm{cL}$ peut être considérée comme un premier pas vers le calcul de la stabilité de la pente, en admettant $\Phi=0$, et les essais de Collin à la boite de cisaillement sont une première tentative pour mesurer la résistance des argiles non drainées. Toutefois, Collin lui-même attachait peu d'importance au calcul et considérait les essais comme une démonstration des effets de l'eau plutôt qu'une dètermination pratique de la résistance au cisaillement. De plus il n'a jamais envisagé la possibilité d'essais sur échantillons intacts ou la détermination de la résistance au cisaillement par le calcul de la stabilité de glissements réels, en fonction des données expérimentales.

En fait, aucun progrès dans cette direction n'a été fait avant de nombreuses années. Mais l'importance pratique des travaux de Collin et de son raisonnement a été immédiatement reconnue par les ingénieurs français.

\section{Biographie}

Alexandre Collin (1808-1890) ancien élève de l'Ecole Polytechnique, Ingénieur des Ponts et Chaussées, a été affecté à des travaux sur le Canal de Bourgogne en 1833. II commença à rédiger son Traité sur les glissements de terrains argileux en 1836. A partir de 1855 il occupa le poste d'Ingénieur en Chef du Loiret à Orléans. II publia des Mémoires sur les injections, les irrigations, I'hydrologie, etc. II prit sa retraite en 1873. (A. W. Skempton, "Alexandre Collin, 1808-1890, Pionnier en mécanique du Sol Trans. Newcomen Soc. 25 (1946), pp. 91-103.)

\section{COMOY - 1875}

\section{Notice sur divers travaux de consolidation de terrains éboulés \\ Annales des Ponts et Chaussées $5^{\circ}$ série - vol. 10 (1875) pp. 8-51.}

Après sa mise à la retraite, Comoy a rédigé ce compte-rendu de travaux de réhabilitation exécutés sous sa direction pendant la période 1856-1866 sur différents glissements (éboulements) de pentes argileuses. Les travaux correspondants comprennent des contreforts dans un talus de chemin de fer et une tranchée et sur la face aval d'une digue en terre, et un mur de soutènement au pied d'une tranchée. Mais les cas les plus intéressants sont ceux relatifs à des travaux de drainage.

Dans I'hiver 1857-1858, des pluies exceptionnelles réactivèrent des glissements anciens, sur les pentes de la vallée de l'Allier inclinées à 9 et $10^{\circ}$, près de Vichy, dans des argiles tertiaires. Ces mouvements s'étendaient sur une longueur de 150 à $200 \mathrm{~m}$ le long de la ligne de plus grande pente suivant des surfaces de glissement typiques, de 3 à $7 \mathrm{~m}$ de profondeur. Dans cinq de ces glissements une seule tranchée a été creusée au centre de la masse en mouvement. Chaque tranchée avait une largeur de $1 \mathrm{~m}$ à la base et était remplie de gravier ou de pierres cassées sur une épaisseur de $2 \mathrm{~m}$; le reste étant comblé en terre. Dans un sixième glissement, d'une largeur exceptionnelle, quatre de ces drains transversaux furent exécutés à $50 \mathrm{~m}$ l'un de l'autre débouchant dans un drain longitudinal près du pied de la pente. Ces tranchées étaient poussées au-dessous de la surface de glissement et là, où, dans un cas particulier, la profondeur dépassait $6 \mathrm{~m}$ sur une longueur de plus de $60 \mathrm{~m}$, on construisit une galerie. Ces travaux furent exécutés pendant les années 1857-1862.

Les mêmes pluies ont provoqué un mouvement dans un glissement ancien (1825) dans la pente d'une colline au-dessus du Canal de Roanne à Digoin près d'Avrilly, dans la vallée de la Loire. Là, quatre tranchées, suivant la plus grande pente, furent creusées jusqu'à la surface de glissement (dans ce cas avec une profondeur maximale de $7 \mathrm{~m}$ ), le fond de chacune étant rempli de gravier sur une hauteur de $2 \mathrm{~m}$. Un drain longitudinal fut également prévu, de $3 \mathrm{~m}$ de profondeur et $2 \mathrm{~m}$ de large, mais les quatre tranchées suivant la ligne de la plus grande pente furent laissées ouvertes au-dessus du remplissage de gravier. Depuis l'achèvement de ce travail en 1857, aucun mouvement ne se produisit jusqu'au moment où Comoy écrivit son mémoire en novembre 1874 .

Le troisième cas est un glissement sur la voie ferrée Bayonne-Irun près de Biarritz, intéressant un remblai établi sur une pente. La surface de glissement, qui allait jusqu'à $13 \mathrm{~m}$ de profondeur, ne put être atteinte par des tranchées; néanmoins, les ingénieurs estimèrent qu'une stabilité suffisante pourrait être obtenu grâce à deux tranchées drainantes, à $30 \mathrm{~m}$ l'une de l'autre, remplies sur environ $3 \mathrm{~m}$ de matériaux graveleux et sur le reste de la hauteur par de la terre compactée. Le travail, exécuté en 1863-1864, s'avéra tout à fait satisfaisant.

Comoy attribue le succès des mesures appliquées à chacun des trois emplacements en partie à la division de la masse en mouvement et en partie, ou surtout, à l'effet de drainage des "eaux internes " grâce à quoi la cohésion fut établie. II parle avec chaleur des travaux "de notre camarade Collin» pour une meilleure compréhension des glissements d'argile.

\section{Biographie}

- Guillaume Emmanuel Comoy (1803-1885) fut élève de l'Ecole Polytechnique et de l'Ecole des Ponts et Chaussées. II travailla de 1828 à 1856 sur le Canal du Centre et le Canal latéral de la Loire. De 1856 à 1861 il dirigea le Service des travaux de contrôle des crues dans le secteur de la Loire. II rédigea plus d'une douzaine de mémoires. (Annales des Ponts et Chaussées, $6^{\circ}$ série, 10 (1885) 441-457.) 
17 a) SOOY SMITH - 1892

Constructions et Fondations à Chicago

Engineering News, vol. 28 (1892) pp. 343345.

\section{b) SHANKLAND - 1897}

Constructions à squelette en acier à Chicago Min. Proc. Inst. Civ. Eng., vol. 128 (1897) pp. 1-27.

Le centre de Chicago est constitué par une argile, molle, moyennement résistante, de 40 pieds $(12 \mathrm{~m})$ d'épaisseur, qui surmonte une argile plus compacte et qui a à sa partie supérieure une croûte mince provoquée par la dessication et qui est elle-même surmontée par 12 à 14 pieds $(3,7$ a $4,3 \mathrm{~m})$ de sable et remblai. En 1890, lorsque Shankland projetait le Temple Maçonnique de 20 étages (connu plus tard sous le nom de Capitole), les ingénieurs de Chicago avaient appris à limiter la pression sous les fondations de leurs "sky-scrapers" à squelette d'acier à environ 1,5 tonne par pied carré $(0,18 \mathrm{MPa})$ et à prévenir des tassements allant pendant une période de plusieurs années jusqu'à 6 pouces $(15 \mathrm{~cm})$. De plus, même si ce point de vue n'était pas généralement admis, Sooy Smith savait que le * tassement lent et progressif résultait de l'expulsion de l'eau du sol ", mots que l'on trouve dans son mémoire de 1892 et qui font écho à l'affirmation de Telford de 1821 ( $\left.n^{\circ} 11\right)$. Et Shankland, en 1897, dit tout aussi nettement que "le tassement est dû à la compression de l'argile qui a pour effet d'expulser l'eau ».

La première observation de tassement sur une longue période à Chicago et probablement la première en quelque point que ce fut, débuta en 1887 grâce à Sooy Smith lors de la construction de l'Auditorium, et se poursuivit pendant 50 ans (Peck - 1948).

Une autre série d'observations commença en 1890 au début de la construction du Monadnock North Block. dans ce cas grâce à Shankland; et son relevé des tassements du Temple Maçonnique (fig. 13) commencé en mai 1891 peu de temps après le début des fondations fut le premier à être publié, en 1897, avec des observations portant sur 4 ans et demi. Une série complète de nivellements exécutés en 1913 permet de tracer un plan des lignes de niveau montrant le tassement après 22 ans. A ce moment, le tassement, moyen était de l'ordre de 10 pouces $(25 \mathrm{~cm})$ comparé avec les 8 pouces $(20 \mathrm{~cm})$ que Shankland avait prévus dans son étude (Peck op. cit.).

\section{Biographie}

- William Sooy Smith (1830-1916), outre ses services pendant la guerre civile, où il atteignit le grade de Général de Brigade, travailla à partir de 1854 à la construction de voies ferrées et de ponts et depuis 1887 comme l'ingénieur-conseil le plus éminent sur les fondations des immeubles de Chicago.

(Dictionnaire des Biographies Américaines, 1937 (Peck op. cit.).)

Edward C. Shankland (1854-1924), après être sorti de l'Ecole Polytechnique Rensselear, tra-
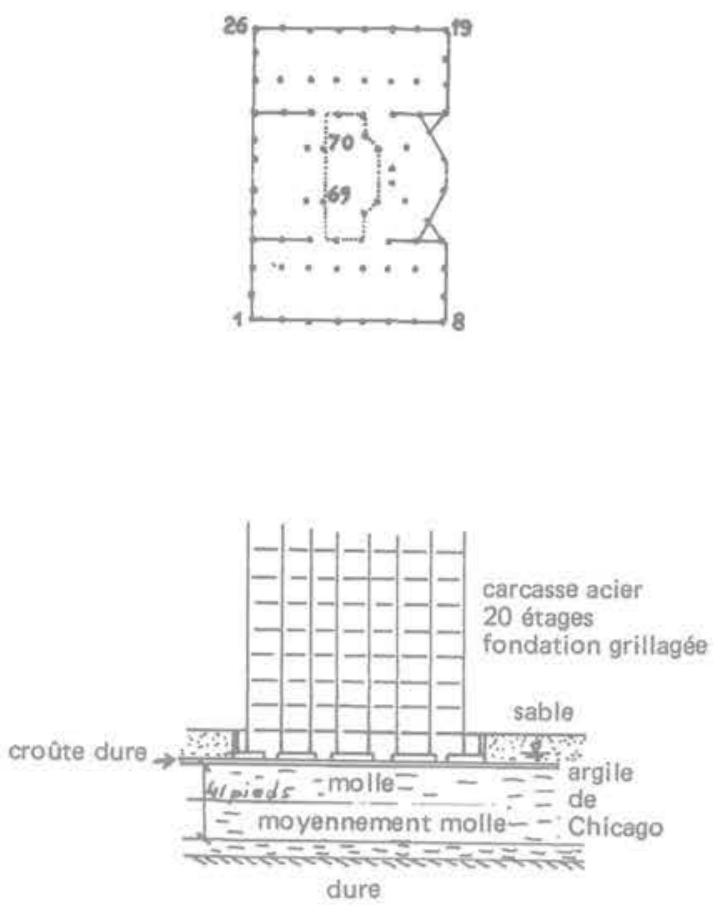

pression sous les fondations : 1,6 t/pied carré

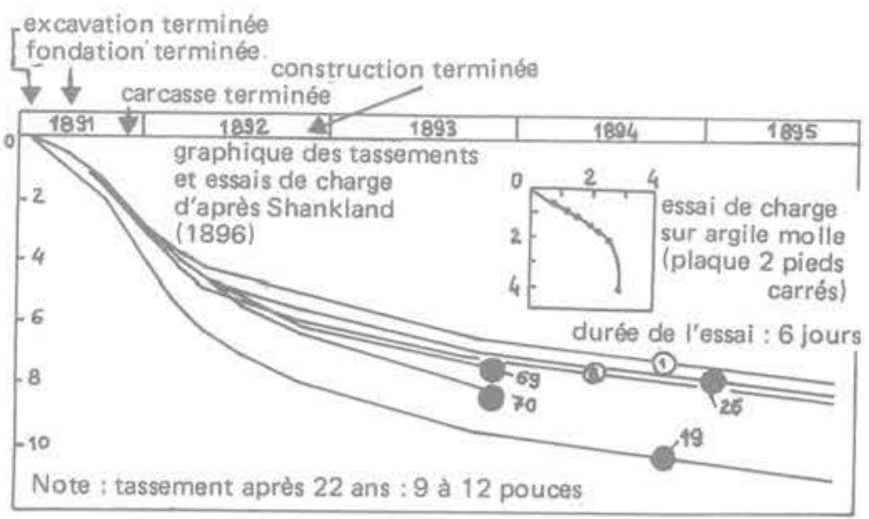

Fig. 13 Temple maçonnique (Capitole), Chicago

vailla aux aménagements du Missouri et, à partir de 1883, à des projets de ponts. II devint ingénieur en 1889 et, de 1894 à 1900, associé au Bureau Burnham and Root à Chicago. Plus tard il s'installa à son compte et on lui doit les projets de nombreux immeubles à structure d'acier de Chicago.

Le Développement de la Construction de Chicago - Presse de I'Université d'lllinois, 1949.

\section{Digues sur fondations perméables}

Lors de l'exécution de digues ou de barrages assis sur des couches de sable relativement profondes, si on ne peut pas réaliser une coupure complète, il est essentiel d'éviter les accidents par percolation et sous-pressions excessives. La "théorie du gradient hydraulique». mise au point au cours des années 1900, a constitué la 


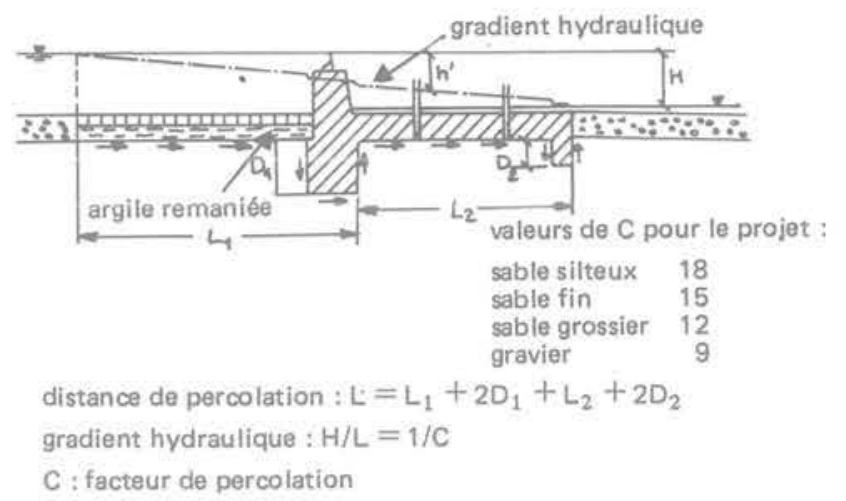

Fig. 14 Théorie du tracé des digues d'après le gradient hydraulique

première solution quantitative de ce problème. Cette theorie (voir fig. 14) se base sur le fait que :

1 La perte de charge $h$ ' en chaque point de l'assise de la fondation ou en un point quelconque de celle-ci est proportionnelle à la longueur du chemin de percolation au point considéré.

2 La sécurité du «facteur de percolation „C, c'est-àdire le rapport entre la distance de percolation et la charge totale $\mathrm{H}$, ne devrait pas être inférieure à des valeurs dépendant de la nature du sol et données en figure 14

Ces valeurs de $C$ ont été publiées par Bligh dans son mémoire : « Barrages, bouchures et digues ", Engineering News vol. 64 (1910) pp. 708-710, dans lequel if expose la pratique courante, telle qu'elle s'était développée aux Indes et en Égypte pendant les 12 dernières années. L'idée d'éviter la formation de renard au moyen de coupures partielles ou de " rideaux étanches", à l'amont et à l'aval de l'assise de la structure hydraulique, remonte à des travaux exécutés aux Indes vers 1830 par Sir Proby Cautley sur le Canal Jumna Est et par Sir Arthur Cotton sur le Système du Delta de Cauvery. Une mesure de sécurité complémentaire a été introduite par le Colonel James Western lorsqu'il construisit un tapis amont imperméable en argile au cours des réparations des chutes de Jaoli, sur le Canal du Gange.

L'utilisation de tapis amont se répandit au cours des vingt années suivantes et devint pratique courante à partir de 1898. Mais jusque-là les projets paraissent avoir été complètement empiriques.

La théorie du gradient hydraulique fut déduite des essais de John Clibborn à Roorkee en 1896-1897 à la suite d'un accident causé par des infiltrations sur la digue Khanki sur la rivière Chenab. Ces expériences montrèrent :

(1) que les infiltrations tendaient à contourner une limite imperméable comme indiqué par les flèches dans la figure 14

(2) que les renards pouvaient commencer dans les sables fins lorsque le gradient hydraulique atteignait 0,1 (avec des valeurs de C de moins de 10 env.) s'il n'y avait pas de rideau aval.

3 Un rideau de moyenne profondeur cause seulement une faible augmentation de la longueur du chemin de percolation mais introduit une marge de sécurité certaine puisqu'il oblige l'eau à percoler vers le haut de la sortie; ceci est nettement une condition plus stable que l'écoulement horizontal tel qu'il se produirait sans rideau. En résumé Clibborn présenta des diagrammes à lignes de gradient hydraulique (ou "pressions de pentes", comme il les nommait), analogues à la figure 14 .

De plus, en 1897 et 1898 , J. S. Beresford fit des essais qui montrèrent pour la première fois les effets d'un filtre inversé en produisant une sécurité complémentaire contre les renards.

Le rapport de Clibborn, écrit en 1897 , fut publié sous le titre «Essais sur la percolation de l'eau à travers le sable de la Rivière Chenab depuis l'emplacement de la digue Khanki», dans son Traité de Roorkee sur l'Engénierie Civile: Travaux d'irrigation aux Indes (Roorkee: Thomason College 1901). Ce rapport parut également, avec une courte note sur les essais de Beresford, dans les Rapports Techniques $n^{\circ} 97$ (Govt of India, 1902). En outre, ce même rapport 97 comportait une note de Beresford sur les observations piézométriques, faites en 1898, dans la fondation de la digue Narora sur le Gange. II utilisa 2 piézomètres comme représenté (fig. 14).

Par une chance extraordinaire, l'assiette de cette digue, dans une baie voisine, s'affaissa seulement quelques jours plus tard. Comme la digue de Khanki elle était fondée sur du sable fin et des deux côtés la rupture se produisit avec des valeurs de C d'environ 9 . Les deux digues furent reconstruites (celle de Khanki terminée en 1898 et celle de Narora en 1900) avec un tapis amont d'argile comportant un mur rideau qui augmenta la valeur de $C$ jusqu'à 15 ou 16 . A la même période, i.e. en 1900, la digue de Jamroa dans le Sind. également assise sur du sable fin, fut construite avec $\mathrm{C}=15$ et se tint de façon tout à fait satisfaisante.

Par ailleurs, la digue Coleron dans l'ensemble du Delta de Cauvery, qui s'était rompue avec $\mathrm{C}=8$ en 1837 (un an après sa construction), fut reconstruite avec $\mathrm{C}=12$ et se comporta bien; mais elle était assise sur du sable grossier. Au contraire, le barrage du Delta du Nil, assis sur un sable fin limoneux, ne devint sûr qu'après remaniement et accroissement de $\mathrm{C}$ jusqu'à 20. Ce travail, entrepris par le Colonel Western, fut terminé au début des années 1890 .

Plus tard, en 1898, en combinant l'expérience acquise en Inde et en Égypte, W. J. Wilson exécuta un magnifique projet pour la fondation du barrage Asyut sur le Nil.

1 II s'assura d'une valeur de $C$ de 20 sous charge normale et de 16 sous la charge maxima possible.

2 II utilisa des palplanches clavées en fer pour les coupures partielles.

3 II plaça un filtre inversé immédiatement après la ligne des palplanches aval et sous l'extrémité de celles-ci et,

4 sur l'avis de Beresford il accrut l'épaisseur du tapis de 2 à $3 \mathrm{~m}$.

Le barrage d'Asyut construit en 1898 à 1902, peut être considéré comme ouvrant une nouvelle époque dans l'étude rationnelle des ouvrages hydrauliques sur des fondations perméables. Après lui vinrent le Barrage Zifta dans le Delta du Nil, construit en 1902-1903, et la Digue Rasul, sur la Rivière Jhelum dans le Punjab, construite en 1899-1901. Tous étaient assis sur du sable fin ou limoneux, avaient des filtres inversés, des tapis aval ou des rideaux de palplanches ainsi que des valeurs de $\mathrm{C}$ supérieures à 16 .

A l'exception de la digue de Jamroa, qui n'a été citée 
que brièvement ici, tous les ouvrages mentionnés sont décrits complètement dans l'ouvrage classique de Buckley: Les travaux d'irrigation en Inde (London : Spon, 1905). II donna aussi une explication claire de la théorie du gradient hydraulique, et un compte-rendu des observations sur les pressions dans les canalisations de Narora.

Tel est l'arrière-plan d'expérience présenté par Bligh en son rapport de 1910. La même année il publia la $2^{\mathrm{e}}$ édition très revue et corrigée de son livre : Projets pratiques de travaux d'irrigation (Londres, Constable, 1910). Celui-ci précise les valeurs de $C$, mais étant un manuel qui couvre l'ensemble de la question, il comprend moins de détails sur les cas particuliers sur lesquels il s'est appuyé pour baser les valeurs utilisées.

La théorie du gradient hydraulique, avec ou sans quelques modifications peu importantes, fut acceptée dans le monde entier. Plus récemment on a introduit des méthodes basées sur les réseaux d'infiltration et la théorie du gradient hydraulique est tombée en désuétude. Mais ceci ne devrait pas réduire le mérite des ingénieurs qui firent les premiers pas dans la compréhension d'une branche importante de la mécanique des sols et qui, dans des conditions difficiles, construisirent d'importants ouvrages pour le bénéfice de millions d'hommes.

\section{Biographies}

- Lt.-Col. John Clibborn (1847-1938) étudia au Trinity College à Dublin, entra à l'Etat-Major de l'Armée des Indes, département d'irrigation, en 1872. II fut directeur du Collège d'Ingénieurs Civils Thomason à Roorkee (1892-1901) (Who-was-Who 1929-1940).

- John Stuart Beresford (1845-1926), après avoir étudié à la Queen's University à Belfast, entra dans le Service des Travaux Publics de I'Inde en 1867. II devint Ingénieur en Chef des Provinces Centrales en 1893 et du Punjab en 1896, puis Inspecteur Général de I'Irrigation 1898-1900. II finit par s'installer à son compte (Who's-Who, 1916-1920).

- William John Wilson (1851-1900), fit ses classes au Collège Royal des Ingénieurs Indiens, à Coopers Hill. II servit dans les travaux publics de 1874-1892. Puis se rendit en Egypte jusqu'à sa mort subite, suite de méningite, en aoút 1900. (Min. Proc. Inst. Civ. Eng., 142 (1900) 383-384.) - Robert Burton Buckley (1847-1927). Etudia à Whitworth, partit en Indes en 1869 et devint Ingénieur en Chef des Travaux Publics (Who's-Who 1916-1928).

- William George Bligh (1846-1923), en service aux Indes au département des Travaux Publics de 1869 à 1889. II fut ensuite transféré aux travaux d'irrigation de Burma et vers 1908 fut nommé à Toronto comme Inspecteur Général du Ministère de I'Intérieur du Canada (Inst. Civ. Eng. records).

\section{IV - Mécanique du sol classique}

Dans les cinquante ans que couvre cette partie de notre exposé, différentes directions de recherche du plus grand intérêt ont été mises en route. II faut citer avant tout les travaux de Rankine, Boussinesq et Résal sur l'analyse des champs de contraintes et la solution correcte présentée par ce dernier du problème des fissures de traction dans les argiles qui a conduit, entre autres, à ce que l'on dénomme la valeur inférieure de la hauteur critique d'une tranchée verticale. Également remarquables sont les essais de Darwin et d'Osborne Reynolds d'où est sorti très clairement le concept de dilatance, les expériences de Darcy sur la perméabilité, les travaux théoriques de Dupuit sur les écoulements d'eau dans le sol et l'introduction par Richardson et Forchheimer de la conception de réseaux d'écoulement.

Un point d'importance spéciale est l'essai fait par Rankine en 1862 d'arriver à une approche unique de la stabilité des pentes et des pressions de terrain pour le sable et l'argile dans des conditions à long terme par une voie simple et très pratique. Et, naturellement, il y a la solution classique de Boussinesq du problème de la répartition des contraintes.

D'autres questions, qui ne peuvent être développées ici, comprennent le cercle des contraintes de Mohr, les méthodes graphiques de détermination des pressions de terrain de Culmann et Engesser, le module de réaction du sous-sol de Winkler et l'analyse des lignes de glissement par Kötter.

En résumé, dès 1910, une grande partie de la mécanique des sols avait été mise au point et le pas en avant suivant devait dépendre d'une meilleure compréhension des propriétés des sols.

\section{RANKINE - 1857}

De la stabilité des terrains meubles Phil. Trans. vol. 147 (1857) pp. 9-27.

A L'époque précise où Coulomb introduisait en mécanique des sols la méthode de l'équilibre limite, Rankine établissait les principes généraux de l'analyse du champ de contraintes (lignes de glissement). Opérant à deux dimensions avec des matériaux graveleux sans cohésion il a donné les équations d'équilibre

$$
\begin{aligned}
& \frac{d \sigma_{x}}{d x}+\frac{d \tau}{d z}=0 \\
& \frac{d \sigma_{z}}{d z}+\frac{d \tau}{d x}=\gamma
\end{aligned}
$$

et la condition de rupture est

$$
\frac{\left(\sigma_{x}-\sigma_{z}\right)^{2}+4 \tau^{2}}{\left(\sigma_{x}+\sigma_{z}\right)^{2}}=\sin ^{2} \Phi
$$

où $\sigma$ et $\tau$ sont les contraintes normales et de cisaillement sur un élément et $\Phi$ "l'angle d'équilibre " défini par la relation de cisaillement

$$
s=\sigma_{n} \operatorname{tg} \Phi
$$

Exprimée en contraintes principales, l'équation (2) devient :

$$
\frac{\sigma_{1}-\sigma_{3}}{\sigma_{1}+\sigma_{3}}=\sin \Phi
$$

et les lignes de glissement ("plans de rupture ") sont 


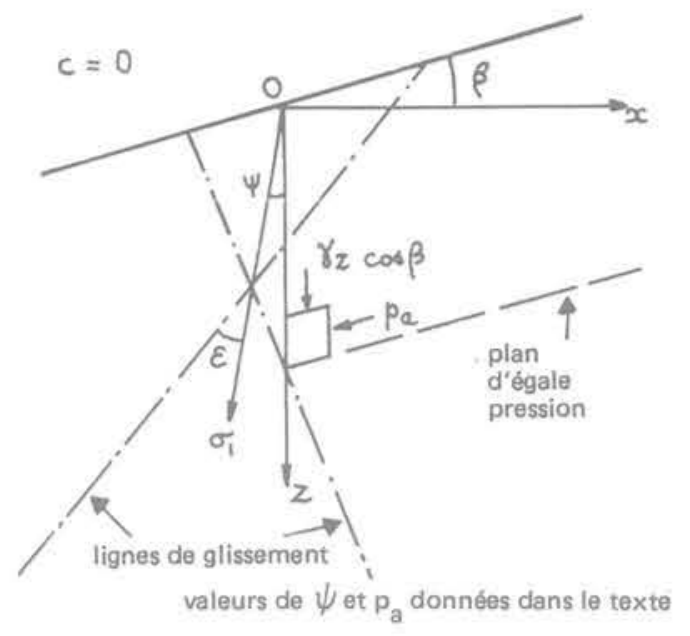

Fig. 15 Rankine, 1857

inclinées à $\pm\left(45-\frac{\Phi}{2}\right)$ par rapport à la direction de $\sigma_{1}$.

Si on considère une masse semi-infinie avec une surface libre inclinée à l'angle $\beta$ sur l'horizontale (fig. 15) un plan à une profondeur constante $z$ est une surface d'égale pression et cette pression est $\gamma z \cos \beta$.

C'est pourquoi la pression conjuguée doit être parallèle à la pente. Rankine montre ensuite que sa valeur minima compatible avec la stabilité est

$$
\mathrm{p}_{\mathrm{a}}=\gamma z \cos \beta\left[\frac{\cos \beta-\sqrt{\cos ^{2} \beta-\cos ^{2} \Phi}}{\cos \beta+\sqrt{\cos ^{2} \beta-\cos ^{2} \Phi}}\right]
$$

et que l'inclinaison de $\sigma_{1}$ sur la verticale, est

$$
\psi=45-\frac{\beta}{2}-\frac{1}{2} \cos ^{-1} \frac{\sin \beta}{\sin \Phi} .
$$

Dans le cas extrême oủ $\beta=\Phi$

$$
p=\gamma z \cos \Phi
$$

et la direction de $\sigma_{1}$ est la bissectrice de l'angle aigu que forment la verticale et la ligne de pente. C'est dire que les lignes de glissement sont soit verticales, soit parallèles à la pente.

Plus généralement si la surface supporte une pression verticale uniforme $q$, on peut substituer $(\gamma z+q)$ à $\gamma z$. Dans le cas où $\beta=0$ on a donc :

$$
\mathrm{p}_{\mathrm{a}}=(\gamma \mathrm{z}+\mathrm{q}) \frac{1-\sin \Phi}{1+\sin \Phi}
$$

et la direction de $\sigma_{1}$ est verticale; les lignes de glissement font donc un angle de $(45-\Phi / 2)$ avec la verticale.

Pour la pression maximale conjuguée, compatible avec la stabilité, les signes des équations précédentes sont inversés; c'est-à-dire que si $\beta=0$

$$
p_{p}=(\gamma z+q) \frac{1+\sin \Phi}{1-\sin \Phi}
$$

et la direction de $\sigma_{1}$ est horizontale.

Sur un plan vertical pénétrant à une profondeur $\mathrm{H}$ sous la surface, l'effort ou résistance totale est obtenue en intégrant $\mathrm{p} \cdot \mathrm{dz}$ de $\mathrm{z}=0$ à $\mathrm{z}=\mathrm{H}$ :

C'est ainsi que pour $\beta=0$

$$
\mathrm{p}_{\mathrm{a}}=\left(\frac{1}{2} \gamma \mathrm{H}^{2}+\mathrm{qH}\right) \frac{1 \pm \sin \Phi}{1 \pm \sin \Phi} .
$$

Enfin Rankine considère l'équilibre des contraintes horizontales sur un plan vertical sous la limite de la fondation. Si la fondation est à une profondeur $D$ sous le sol horizontal et si $q_{f}$ est la pression de fondation qui provoque la rupture, à chaque profondeur $\mathrm{z}$ sous la base, on a

ou $\quad \mathrm{q}_{\mathrm{f}}=\gamma \mathrm{D}\left(\frac{1+\sin \Phi}{1-\sin \Phi}\right)^{2}+\gamma z \frac{4 \sin \Phi}{(1-\sin \Phi)^{2}}$.

II est clair que la valeur minimale se produit pour $z=0$ lorsque

$$
\mathrm{q}_{\mathrm{f}}=\gamma \mathrm{D}\left[\frac{1+\sin \Phi}{1-\sin \Phi}\right]^{2}
$$

Mais nous observons que ceci correspond à une rupture locale à la limite de la fondation. C'est pourquoi Rankine n'a obtenu qu'un champ de contraintes partiel et que sa solution est la même que le cas limite de Poncelet (voir $n^{\circ} 9$ ).

\section{Biographie}

- William John Macquorn Rankine (1820-1872) fit ses études à l'Université d'Edinburgh, remplit les fonctions d'ingénieur civil en Irlande et Ecosse de 1838 à 1855, date à laquelle il fut nommé Professeur Royal de génie civil à Glasgow. F. R. S, auteur de mémoires fondamentaux sur la thermodynamique et la résistance des matériaux, d'ouvrages de fond sur la mécanique appliquée (1858), les machines à vapeur (1859), le génie civil (1862) et les machines (1869). II exerça une influence profonde sur la formation des ingénieurs en Grande-Bretagne (Dictionnaire de Biographie Scientifique, 1975 - Hugh B. Sutherland: Rankine sa vie et son temps. Inst. Civ. Eng. (1973)). Son mémoire "Sur la stabilité des terrains meubles " a été lu à la Société Royale le 19 juin 1856 .

\section{RANKINE - 1862}

\section{Manuel de l'Ingénieur Civil Londres, Griffin and Bohn, 1862.}

Cet ouvrage devint immédiatement un texte fondamental et resta en service avec de faibles modifications pendant au moins 50 ans. Les points particuliers intéressant la mécanique des sols peuvent être résumés comme suit :

1 Les travaux en terre périssent par glissement d'une de leur partie sur une autre. La résistance au cisaillement résulte en général du frottement entre grains et de leur adhésion mutuelle.

2 L'adhésion (ou la cohésion) dans les sols est en général détruite par l'action de l'air, de l'humidité et de la température.

3 Cependant, l'adhésion est utile dans les travaux temporaires en permettant aux faces d'une tranchée de tenir pendant un certain temps à la verticale jusqu'à une certaine profondeur. Cette profondeur dépend de $\mathrm{c} / \gamma$ et va de zéro pour le sable sec, à 3 à 6 pieds ( 1 à $2 \mathrm{~m}$ ) pour de la terre ordinaire (1 à $2 \mathrm{~m}$ ) et 10 à 16 pieds pour de l'argile ( 3 à $5 \mathrm{~m}$ ).

4 La stabilité permanente du sol, due au frottement seul, maintient les faces d'un talus ou d'une tranchée à une pente dont l'inclinaison est l'angle de repos $\Phi$ où $f=\operatorname{tg} \Phi$. C'est ce que l'on nomme l'angle de talus naturel. 
5 La cohésion et le frottement du sol sont si variables que l'ingénieur ne devrait jamais faire confiance à des livres ou à des tables lorsqu'il a la possibilité d'obtenir les renseignements nécessaires, soit par l'observation de travaux en terre dans le même matériau, ou par l'expérimentation.

6 Néanmoins, à titre de guide, Rankine a établi des tables donnant $\Phi$ pour différents types de sol. Il y a une faute d'impression $\left(21^{\circ}\right.$ au lieu de $\left.31^{\circ}\right)$ pour la limite inférieure des sables, mais, sans m'y attacher, j'ai présenté dans le tableau 4 quelques valeurs typiques. On verra que pour les argiles, ces "angles de repos" correspondent à la stabilité à long terme ou «permanente $"$ avec $\mathrm{c}=0$.

7 En général on peut faire confiance à la cohésion de la roche et on peut donner une pente raide aux pentes des excavations taillées dans celles-ci. Mais les schistes sont susceptibles de se ramollir sous l'effet de I'hygrométrie et il est des schistes qui sont à peine plus résistants que de la terre et qui nécessitent des pentes de $1: 1$ ou $1,5: 1$.

$8 \quad$ II reprend (voir $n^{\circ} 19$ ) les expressions auxquelles il était déjà arrivé dans son Mémoire de 1857 de poussée, de butée et de charge portante.

9 II introduit en outre la conception d'une unité équivalant de masse $\gamma_{\theta}$ telle que

$$
\begin{gathered}
P_{a}=\frac{1}{2} H^{2} \gamma_{e} \\
\gamma_{e}=\gamma K_{a} .
\end{gathered}
$$

ou

On trouvera table 4 quelques valeurs de $\gamma_{e}$ pour le cas d'un mur vertical et d'un remblai horizontal.

10 En supplément à son Mémoire de 1857, Rankine donne l'expression de la traction limite $T$, qui peut être appliquée à la chaîne horizontale d'une poutre d'ancrage. Si le haut et le bas de la poutre de l'ancre sont à des profondeurs $D_{1}$ et $D_{2}$ au-dessous du niveau du sol, on a

$$
T=\frac{1}{2} \gamma\left(D_{2}^{2}-D_{1}^{2}\right) \frac{4 \sin \Phi}{\cos \Phi} .
$$

Ceci résulte directement de l'intégration de $\left(\mathrm{p}_{\mathrm{p}}\right.$ $\left.\mathrm{p}_{\mathrm{a}}\right) \mathrm{dz}$.

11 Les murs de soutènement devraient être étudiés de manière que :

a) la résultante passe par le tiers central de la base, pour éviter des tractions ou des sous-pressions au talon;

b) l'obliquité de la résultante ne doit pas dépasser $\Phi_{1}$, angle de frottement entre la fondation et le terrain sous-jacent et Rankine donne des valeurs de $\Phi_{1}$ égales à $18^{\circ}$ pour de l'argile humide et à $27^{\circ}$ pour de I'argile sèche;

c) la pression maxima de fondation ne doit pas dépasser la charge portante admissible.

12 Les fondations de murs et de constructions peuvent être classées comme assises sur a) de la roche, b) de la terre résistante, comme du sable, c) de la terre meuble. Pour le rocher sain, on peut utiliser des charges de $10 \mathrm{t}$ /pied carré $(1,2 \mathrm{MPa})$, bien que dans certains grès tendres il ne faille pas dépasser $2 t / p i e d$ carré $(0,25 \mathrm{MPa})$.

Dans ur terrain résistant les fondations devraient être descendues jusque 3 et 4 pieds $(1 \mathrm{~m}$ ) sous le niveau du sol, pour éviter la désintégration par le gel et la sécheresse; les charges sont en général limitées à environ 1,5 t/pied carré $(0,19 \mathrm{MPa})$. Lorsque le terrain est meuble on peut utiliser une plate-forme en bois ou une dalle en béton pour répartir la charge autant que possible, mais en général on utilise les fondations sur pieux. Celles-ci peuvent constituer des groupes de pieux courts, de 6 à 12 pieds de long ( 2 à $4 \mathrm{~m}$ ), battus aussi près que possible l'un de l'autre, en réalité pour abaisser d'autant le niveau d'application de la charge. Dans d'autres cas les pieux sont écartés les uns des autres et chacun supporte sa part de la charge. Les pieux peuvent être battus à travers les couches molles jusqu'à un matériau résistant ou, si cela n'est pas possible, elles doivent transmettre leur charge par frottement dans les couches molles.

13 Les remblais, assis sur des terrains peu résistants, peuvent comporter des pentes dont les angles ne dépassent pas l'angle de repos $\Phi_{1}$ du sol. On peut aussi excaver le sol jusqu'à une profondeur $h_{1}$ et remplir l'excavation par du matériau, $h_{1}$ étant déterminé par l'expression

$$
h_{1}\left[k^{2} \gamma_{1}-\gamma\right]=\gamma h
$$

où $\gamma_{1}$ et $\gamma$ sont les poids volumiques du sol et du remblai, $h$ la hauteur du remblai et $k=$ $(1+\sin \Phi) /(1-\sin \Phi)$. Les pentes en tranchées seront inclinées à $\Phi_{1}$ et celles de remblais en fonction da ce qui a été dit ci-dessus.

14 La meilleure méthode pour s'assurer de la nature du sol est de forer un ou plusieurs puits combinés avec des sondages qui, pour des tranchées ou des tunnels, doivent être à 200 ou $300 \mathrm{~m}$ l'un de l'autre. Ces sondages ne procurent que des échantillons perturbés (ou fragmentés) ou ramollis par l'eau courante, mais ils montreront s'il y a suffisamment de changements dans les couches pour obliger à creuser des puits supplémentaires.

Rankine a été critiqué dans les temps modernes pour n'avoir pas tenu compte de la cohésion et, du fait de son autorité, d'avoir perpétué une théorie parfois trop simplifiée. Il était naturellement au courant de l'analyse des pressions de terrain en c, $\Phi$ mais l'estimait douteuse pour les applications pratiques par défaut de données expérimentales. En 1862 cette remarque était justifiée. Ni lui ni personne autre ne pouvait donner des chiffres valables à la fois pour $\mathrm{c}$ et $\Phi$. Au lieu d'adopter ce que l'on pourrait appeler une approche semiempirique, basée sur l'observation des pentes permanentes dans l'argile et d'utiliser des valeurs de $\Phi$ déduites des observations et basées sur l'hypothèse $c=0$, il reconnut clairement que cette méthode ne pouvait être appliquée à des problèmes à court terme dans l'argile, mais on peut se demander si le bien-fondé de l'hypothèse $\mathrm{c}=0$ ne peut pas être remis en question.

Pour les sols sans cohésion on peut critiquer le fait qu'il néglige le frottement du mur. II a également négligé de traiter la pression hydrostatique due aux nappes phréatiques, bien que, comme on le verra dans le Mémoire de Baker ( $\left.n^{\circ} 24\right)$, ceci ne présentait pas de difficultés dans les sols perméables.

Rankine introduit une courte description, des outils de forage et de prélèvement d'échantillons. Elle est basée sur le compte-rendu détaillé de W. D. Haskoll: Le Guide du forage à l'attention de l'ingénieur-adjoint de voie ferrée (Londres, 1846). Mais la technique était déjà bien établie à l'époque et les compte-rendus de forages de reconnaissance remontent en Angleterre à 1770 . 


\section{Tableau 4}

Quelques valeurs types de poids volumique $\gamma$ (livre/pied cube) et d'angle de repos $\Phi 1829$ volumique long terme) avec les valeurs correspondantes des coefficients de pression de terrain $K_{a}$ et $K_{p}$ (pour $\beta=0$ et des murs verticaux) et le poids volumique équivalent $\gamma_{\mathrm{e}}=\gamma \cdot \mathrm{K}_{\mathrm{a}}$ d'après Rankine, 1862

\begin{tabular}{|l|c|c|l|l|l|l|}
\hline & $\gamma(\mathrm{lb} / \mathrm{cu} \cdot \mathrm{ft})$ & $\Phi^{\circ}$ & $\cot \Phi$ & $\mathrm{K}_{\mathrm{a}}$ & $\gamma_{\mathrm{e}}$ & $\mathrm{K}_{\mathrm{p}}$ \\
\hline Argile humide & 120 & 16 & 3,5 & 0,57 & 68 & 1,8 \\
Argile sèche & 130 & 27 & 2 & 0,38 & 50 & 2,6 \\
Sable & 100 & 33 & 1,5 & 0,29 & 29 & 3,5 \\
Gravier & 100 & 38 & 1,25 & 0,23 & 23 & 4,3 \\
\hline Briques & 112 & & & & & \\
Maçonnerie & 130 & & & & & \\
\hline
\end{tabular}

\section{DARCY - 1856}

\section{Les Fontaines Publiques de la Ville de Dijon} Paris, Dalmont, 1856.

James Simpson avait introduit des filtres à sable, de granulométrie déterminée, à l'usine de purification d'eau de Chelsea en 1829 et ceux-ci furent adoptés dans une demi-douzaine de villes en Grande-Bretagne et en France dans les vingt ans qui suivirent. Darcy donne des détails sur ces installations, mais se rend compte que les données relatives à leurs performances ne permettaient pas de déduire une loi générale sur l'écoulement de l'eau dans le sable. II décida donc d'aborder le sujet expérimentalement et réalisa 36 essais à Dijon entre octobre 1855 et février 1856.

Son appareillage comprenait un tube vertical de $35 \mathrm{~cm}$ de diamètre équipé à sa base d'une grille à mailles fines supportant un lit de sable, et de manomètres à mercure permettant de lire la pression de l'eau au-dessus et au-dessous du sable. Avec $58 \mathrm{~cm}$ de sable dans le tube, l'écoulement était mesuré sous des charges différentielles comprises entre $1 \mathrm{~m}$ et $10 \mathrm{~m}$, chaque essai durant environ 20 minutes. Les essais étaient répétés avec des épaisseurs de 110 à $170 \mathrm{~cm}$ de sable.

En se rapportant à la figure 16, si les hauteurs piézométriques au-dessus et au-dessous d'une couche de sable d'épaisseur $L$ et de surface $A$ sont respectivement $(h+L)$ et $h_{0}$, et si $Q / A$ est la quantité d'eau qui s'écoule par unité de surface (c'est-à-dire la rapidité de l'écoulement v), Darcy a trouvé que pour toutes les valeurs de $h, h_{0}$ et $L$ les résultats de ses essais satisfaisaient l'expression

$$
\frac{Q}{A}=v=k \frac{(h+L)-h_{0}}{L}=k \frac{\Delta h}{L}
$$

oủ $k$ est une constante qu'il définit comme «un coefficient dépendant de la perméabilité de la couche de sable *.

L'équation (1) ci-dessus est connue comme la loi de Darcy. Elle est analogue à la loi de l'écoulement dans les tubes capillaires établie par Poiseuille en 1841.

Dans ses essais, Darcy utilisait un sable de granulométrie moyenne avec une porosité de $38 \%$. La valeur de $k$ était environ $0,03 \mathrm{~cm} / \mathrm{s}$.

Dans chaque essai, h et $h_{0}$ étaient maintenus constants pendant la mesure de Q. Mais Darcy a aussi considéré le cas où une certaine quantité d'eau est placée sur une

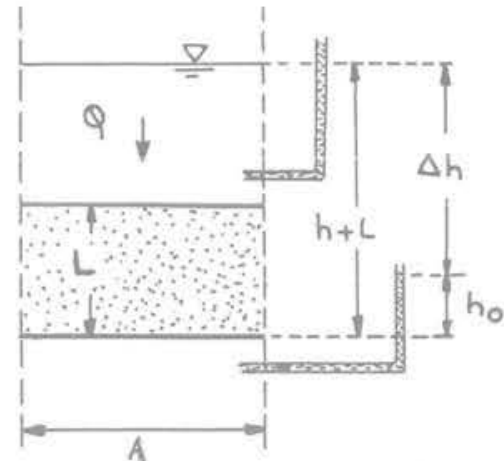

Les essais à charge constante montrent :

$$
\frac{Q}{A}=v=k \frac{(h+L)-h_{0}}{L}=k \frac{\Delta h}{L}
$$

oủ $k$ est le coefficient de perméabilité à charge variable, avec $h_{0}$ constant on a :

$$
\begin{gathered}
v=-\frac{d h}{d t} \\
\log _{e} \frac{\Delta h_{1}}{\Delta h_{2}}=k \frac{t_{2}-t_{1}}{L} .
\end{gathered}
$$

Fig. 16 Darcy, 1856

couche de sable à travers laquelle elle percole sous le seul effet de la gravité. La hauteur d'eau h diminuera avec le temps, et la rapidité de l'écoulement à chaque instant $\left(h_{0}\right.$ étant constant) sera

$$
v=-\frac{d h}{d t} \text {. }
$$

En combinant cette expression avec l'équation (1) on voit que

$$
\log \frac{\Delta \mathrm{h}_{1}}{\Delta \mathrm{h}_{2}}=\mathrm{k} \frac{\mathrm{t}_{2}-\mathrm{t}_{1}}{\mathrm{~L}}
$$

qui est l'équation de l'écoulement à « charge décroissanten. Darcy l'a confirmé expérimentalement.

\section{Biographie}

- Henri Philibert Gaspard Darcy (1803-1858), Inspecteur Général des Ponts et Chaussées, a étudié et dirigé la construction de l'approvisionnement en eau de la Ville de Dijon (1834-1842) et la partie de la voie ferrée Paris-Lyon traversant la Cóte d'Or (1842-1848). II devint ensuite Ingénieur en Chef des Services Municipaux de la Ville de Paris. II retourna à Dijon en 1855 et se consacra entièrement aux recherches hydrauliques. (Dictionnaire Biographie Française, 1970.)

\section{DUPUIT - 1863}

Etudes théoriques et pratiques sur le mouvement des eaux dans les canaux découverts et à travers les terrains perméables

Paris, Dunod, 1863.

Dans cette édition qui complète un travail antérieur 
(1848), Dupuit traite pour la première fois d'un écoulement permanent, sous l'effet de la gravité, d'une nappe dans des terrains perméables. II fait I'hypothèse simplificatrice que le gradient hydraulique est pour chaque section verticale:

a) égal à la pente de la surface libre de la nappe.

b) constant sur toute la hauteur de cette section.

Ces approximations, il le reconnaît, ne sont valables que pour de faibles gradients. L'écoulement dans une couche perméable surmontant une limite horizontale imperméable, peut s'exprimer comme suit par unité de largeur

$$
q=-k h \frac{d h}{d x}
$$

où $\mathrm{h}$ est la distance de la surface libre à la limite étanche et $\mathrm{k}$ le coefficient de perméabilité.

Dupuit aborde tout d'abord l'écoulement à deux dimensions. Si $h_{0}$ est la profondeur pour $x=0$ et $h$ la profondeur $\left(h>h_{0}\right)$ à la distance $x$ de l'origine, l'intégration de l'équation (1) donne

$$
q=k \frac{h^{2}-h_{0}^{2}}{2 x}
$$

Ceci peut être appliqué directement à un talus à faces verticales comme un batardeau. Si $h_{w}$ est la hauteur d'eau en aval (où $\mathrm{x}=0$ ) et $\mathrm{H}$ la hauteur d'eau en amont, on a

$$
q=k \frac{H^{2}-h_{w}^{2}}{2 L}
$$

où $L$ est la largeur de la digue. De plus, les équations (2) et (3) montrent de suite que la surface libre à l'intérieur de la digue est

$$
h^{2}=h_{w}^{2}+\frac{X}{L}\left(H^{2}-h_{w}^{2}\right) .
$$

Pour analyser l'écoulement tridimentionnel vers un puits équipé d'une pompe, Dupuit suppose le puits, de rayon $r_{w}$, placé au centre d'une île de sable de rayon $A$ à bords verticaux au-delà desquels le niveau est maintenu à la profondeur $\mathrm{H}$. II montre ensuite que l'écoulement total vers le puits est :

$$
Q=k \frac{\pi\left(H^{2}-h_{w}^{2}\right)}{\log ^{2} / r_{w}}
$$

où $h_{w}$ est la profondeur de l'eau dans le puits et que l'équation de la surface libre est

$$
h^{2}=h_{w}^{2}+\left(H^{2}-h_{w}^{2}\right) \frac{\log r / r_{w}}{\log { }^{n} / r_{w}} \text {. }
$$

De même l'écoulement vers un puits artésien à partir d'un aquifère horizontal de dimensions limitées, d'épaisseur D, est

$$
Q=k \frac{2 \pi D\left(H-h_{w}\right)}{\log { }^{2} / r_{w}}
$$

où $\mathrm{H}$ et $\mathrm{h}_{\mathrm{w}}$ sont les niveaux piézométriques à la limite extérieure et au puits. Ces équations s'appliquent à un puits pénétrant toute l'épaisseur du sable.

Pour les recherches ultérieures sur les infiltrations vers le puits, voir Hall (1954). Mais on peut dès maintenant attirer l'attention sur les quatre points suivants :

1 L'étendue de la couche perméable est probablement d'une dimension presque illimitée par rapport à sa profondeur, mais on ne peut lui donner une valeur infinie. Cette difficulté a été résolue en principe par Adolphe Thiem, lequel, en 1870 , se rendit compte qu'au-delà d'une certaine distance, le rabattement était négligeable ou nul, du fait que le remplissage du puits par la nappe phréatique ou la pluie tend à compenser la quantité drainée par le puits, si bien que $\mathrm{H}$ peut être pris comme la profondeur initiale de l'eau à une certaine distance, finie, la valeur de R étant considérée comme le rayon d'influence.

2 Pour des gradients raides les surfaces libres résultant des équations (4) et (6) sont des approximations grossières, mais, fait surprenant, les équations (3) et (5) sont des expressions mathématiques exactes du volume de l'écoulement. Ce point est expliqué par P. Y. Polubarinova-Kochina dans son Traité du mouvement de la nappe phréatique, traduit par R. de Weist (Princeton, 1962).

3 Le problème de l'écoulement vers une ligne ou un groupe de puits, important dans le processus des rabattements de nappes, a été traité par Philippe Forchiheimer en 1898 et dans des Mémoires ultérieurs.

4 L'emploi d'essais de pompage in-situ pour déterminer la perméabilité, utilisant l'équation (5) et utilisant des observations dans des piézomètres ou des tubes plantés dans le sol, à une certaine distance du puits, est due à Günther Thiem en 1906.

\section{Biographie}

- Arsène Jules Etienne Juvénal Dupuit (1804-1866) est entré aux Ponts et Chaussées en 1824 après être passé par l'Ecole Polytechnique. II a été Ingénieur en Chef du Maine-et-Loire à partir de 1842 et de la Ville de Paris à partir de 1850. II est l'auteur de différents Mémoires et de livres sur des aspects divers de l'art de l'ingénieur civil. (Dictionnaire Biographie Française, 1970).

\section{BOUSSINESQ - 1876 et 1885}

a) Essai théorique sur l'équilibre d'élasticité des massifs pulvérulents et sur la poussée des terres sans cohésion

Mémoires de l'Académie Royale de Belgique - vol. 40 (1876) p. 180. Réimprimé avec un titre légèrement différent à Paris chez Gauthier-Villars, 1876.

b) Sur l'intégration par approximation successives d'une équation ... dont dépendent les pressions intérieures d'un massif de sable à l'état ébouleux

Dans I'Application des Potentiels, Paris 1885 (voir $n^{\circ} 27$ ) pp. 705-712.

L'analyse de Rankine des contraintes dans une masse semi-infinie de sol sans cohésion est correcte aussi loin qu'elle va, mais est très limitée quand on cherche à I'appliquer à des murs de soutènement, du fait qu'elle ne tient pas compte du frottement du mur comme d'une variable indépendante. En réalité, elle admet que la pression de terrain agit sur le mur dans une direction parallèle à la surface libre. Ce problème des murs de soutènement, analysé en fonction des contraintes en place, a attiré l'attention de Maurice Lévy en 1867 et de Barré de Saint-Venant en 1870 (Heyman, 1972) et des progrès importants ont été faits par Boussinesq. Ses recherches débutèrent en 1873 et il présenta son Essai à l'Académie Royale des Sciences de Belgique en juin 1874. II fut publié deux ans après. 
Boussinesq commence en considérant une masse de sable sec avec une surface inclinée, en état d'équilibre élastique sous son propre poids. Son analyse est basée sur l'hypothèse d'une compressibilité négligeable et d'un module de cisaillement $\mu$ proportionnel à la pression moyenne. Je ne reprendrai pas cette partie de son Mémoire, si ce n'est pour signaler que dans son introduction, Boussinesq indique que l'on peut négliger la pression atmosphérique «du fait qu'elle agit dans toutes les directions à l'intérieur de la masse et autour de chaque grain... Elle n'a donc aucune influence sur leur action mutuelle et, par conséquent, ne modifie pas les forces supplémentaires normales et tangentielles que les contacts entre grains produisent sur l'unité de surface de chaque élément. Seules, ces forces supplémentaires doivent être prises en compte $"$. Nous avons ici une expression claire, même si elle est restrictive, du principe des contraintes effectives.

Passant au problème de la pression de terrain, Boussinesq admet que le mouvement latéral a été suffisant pour mettre la masse de sable en état d'équilibre plastique ou limite. La difficulté est alors de combiner les équations (1) et (2) de Rankine avec la condition aux limites $\tau=\sigma_{\mathrm{n}} \operatorname{tg} \delta$ sur le plan représentant la partie arrière du mur de soutènement.

Boussinesq prend le cas général d'une paroi inclinée et d'un remblai en pente et réussit à trouver une solution approchée pour la pression de terrain agissant sous un angle $\delta$ par rapport à la normale à la paroi. Pour la simplicité, si on considère un mur vertical et un remblai horizontal (fig. 17), il y a une discontinuité dans le champ de contraintes entre l'état défini par Rankine dans la zone $O A Q$ et le coin $O M Q$ et les lignes de glissement dans le coin doivent être incurvées.

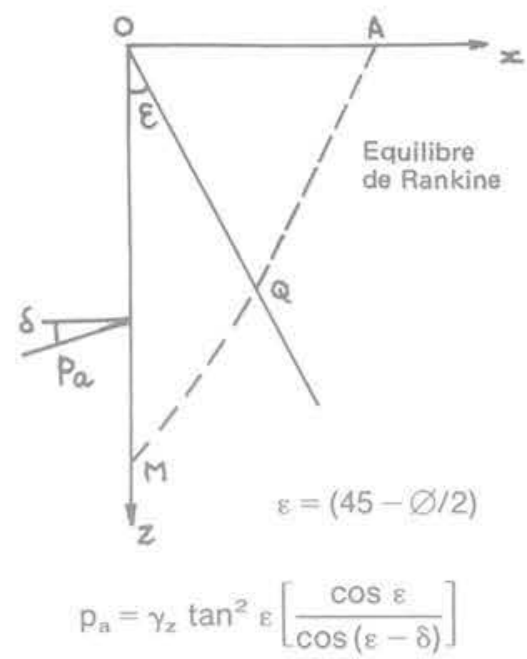

Fig. 17 Boussinesq, 1876
Écrivant que la pression de terrain à toute profondeur sur le plan $x=0$ a pour expression

$$
\mathrm{p}=\gamma \mathrm{z} \cdot \mathrm{K}
$$

it trouve en première approximation

$$
\text { ou } \quad \begin{aligned}
\mathrm{k} \cos \delta & =\mathrm{a}^{2} \frac{1}{1+\mathrm{a} \operatorname{tg} \delta} \\
\text { où } \quad \mathrm{a} & =\operatorname{tg}\left(\frac{\pi}{4}-\frac{\Phi}{2}\right)=\operatorname{tg} \varepsilon .
\end{aligned}
$$$$
K=\operatorname{tg}^{2} \varepsilon \frac{\cos \varepsilon}{\cos (\varepsilon-\delta)}
$$

Les valeurs de $K \cos \delta$ tirées de l'équation (2) figurent au tableau 5.

Boussinesq reprit ce problème plusieurs fois et, finalement, dans un chapitre supplémentaire de I'Application des Potentiels (1885), il obtint une seconde approximation laquelle, pour des parois verticales et un remblai horizontal, peut être mise sous la forme:

$$
\mathrm{K} \cos \delta=\mathrm{a}^{2} \frac{1}{1+\mathrm{a} \operatorname{tg} \delta} \mathrm{C}
$$

oủ

$$
\mathrm{C}=1+\frac{1}{\sin \Phi}\left[\frac{\mathrm{a} \operatorname{tg} \delta}{1+\mathrm{atg} \delta}\right]^{2} \log \frac{4}{\ell} .
$$

II pensait que cette expression correspondait à une limite supérieure mais les résultats (table 5) sont numériquement presque identiques aux meilleures solutions de l'analyse limite, disponibles actuellement (W. F. Chen : Analyse limite et plasticité des sols) Amsterdam (1975) et sont légèrement inférieures aux valeurs du champ de contraintes obtenues par Caquot et Kerisel (Traité de Mécanique des sols, Paris 1949), et par V. V. Sokolovski (Statique des sols, traduit par D. H. Jones and A. N. Schofield, Londres, 1960).

\section{Biographie}

- Joseph Valentin Boussinesq (1842-1929) un autodidacte en mathématiques appliquées, puissant et original. II commença sa carrière comme simple maitre d'école pour arriver membre de l'Académie des Sciences, Professeur à I'Université de Lille en 1873 et à la Sorbonne en 1886. (Dictionnaire de Biographie Scientifique (1970) Armand Mayer: Géotechnique 4 (1954) 3-5.

Valeurs de $\mathrm{K} \cos \delta$ où $\mathrm{P}_{\mathrm{a}}=\frac{1}{2} \gamma \mathrm{H}^{2} \mathrm{~K}$ cas du mur vertical et du remblai horizontal

\begin{tabular}{|c|c|c|c|c|c|}
\hline \multirow{2}{*}{$\Phi^{\circ}$} & $\delta=0$ & \multicolumn{4}{|c|}{$\delta=\varnothing$} \\
\cline { 4 - 5 } & & & \multicolumn{2}{|c|}{ Boussinesq } & $\begin{array}{c}\text { Caquot } \\
\text { et } \\
\text { Kerisel }\end{array}$ \\
\cline { 4 - 5 } & Rankine & Coulomb & 1876 & 1885 & 0,267 \\
\hline 30 & 0,333 & 0,257 & 0,250 & 0,262 & 0,213 \\
35 & 0,271 & 0,205 & 0,199 & 0,208 & 0,168 \\
40 & 0,217 & 0,161 & 0,156 & 0,164 & 0,131 \\
45 & 0,172 & 0,125 & 0,121 & 0,127 & 0 \\
\hline
\end{tabular}




\section{La véritable pression latérale dans les ouvrages en terre}

Min. Proc. Inst. Civ. Eng. vol. 65 (1881) pp. 140-186.

Baker adopte la théorie classique de la pression de terrain, dans l'hypothèse $\mathrm{c}=0, \delta=0$, en exprimant les résultats en termes de poids volumique équivalent (voir $n^{\circ} 20$ ) et il montre par l'application de cette théorie que la poussée latérale et le moment sur des murs expérimentaux essayés par Hope $\left(n^{\circ} 10\right)$ et d'autres, sont surestimés. Curieusement, il ne tient pas compte du frottement sur le mur, mais dans une correspondance relative à ce mémoire (publiée dans le même volume), Flamant et Boussinesq tiennent compte de cet élément et montrent qu'il permet de résoudre les différences.

Baker décrit ensuite quelques-uns des murs de soutènement du Métropolitain de Londres. Un mur de 23 pieds de haut $(7 \mathrm{~m})$ retenant du sable sec et calculé pour $\gamma_{\mathrm{e}}=20$ livres/pied cube $\left(3,2 \mathrm{kN} / \mathrm{m}^{3}\right)$ resta stable alors qu'il aurait dû se rompre, du fait que l'on avait $\Phi=37^{\circ}$ (ici aussi nous notons que le frottement sur le mur permet de rendre compte de certaines anomalies). En un autre emplacement où des apports de terre et de gravier de 20 pieds d'épaisseur $(6 \mathrm{~m})$ surmontaient I'argile de Londres, un mur de 30 pieds de haut $(9 \mathrm{~m})$ se rompit par glissement et rotation. II fut reconstruit avec une section un peu plus large et une fondation plus profonde pour tenir environ 55 livres/pied cube $\left(8,8 \mathrm{kN} / \mathrm{m}^{3}\right)$ et resta stable. Un deuxième mur dans le voisinage, prévu pour une charge unitaire équivalente. plutôt plus importante, bascula légèrement et se déplaça vers l'avant, mais il y avait, pas loin derrière le mur, une conduite qui fuyait.

Une vingtaine de murs de quai sont également décrits. Là où la pression équivalente utilisée pour le calcul est indiquée, elle varie depuis une valeur exceptionnellement basse de 30 livres/pied cube $\left(4,8 \mathrm{kN} / \mathrm{m}_{3}\right)$ à une valeur qui paraît plus normale de 50 à 60 livres/pied cube ou même 80 livres/pied cube ( 8 à 9,6 ou même $12,8 \mathrm{kN} / \mathrm{m}^{3}$ ) dans le cas d'un mur adossé à une argile très plastique. Un seul de ces murs se rompit par basculement, mais plusieurs furent détruits par glissement vers l'avant sur l'argile. Au moins deux de ceux-ci paraissent avoir été calculés avec un angle de frottement à la base de $27^{\circ}$, c'est-à-dire un facteur de sécurité de 1.0 par rapport à la valeur la plus élevée donnée par Rankine pour $\Phi$ dans l'argile, et, en général, les fondations étaient trop superficielles pour développer une butée appréciable; un élément rappelé avec insistance par Poncelet $\left(n^{\circ} 9\right)$, mais, semble-t-il. oublié par certains ingénieurs de la fin du $19^{\circ}$ siècle.

Prenant une vue d'ensemble de tous les essais en piace, Baker conclut que la théorie classique n'a qu'une utilité pratique limitée. Mais cette opinion ne paraît pas entièrement justifiée, et je dirais volontiers que si l'on avait dans tous les cas pris en compte la sécurité en cas de glissement, avec les valeurs de pression de terrain indiquées par la théorie de Rankine, et des angles de frottement internes raisonnables, ceux-ci auraient permis un calcul avec une bonne approximation de la stabilité à long terme dans l'argile.

Un des points les plus intéressants dans le mémoire se présente à l'occasion des écluses où le niveau de l'eau devant le mur est souvent beaucoup plus bas que celui derrière celui-ci (fig. 18). Ici Baker indique que sous le niveau de la nappe phréatique la pression totale est la

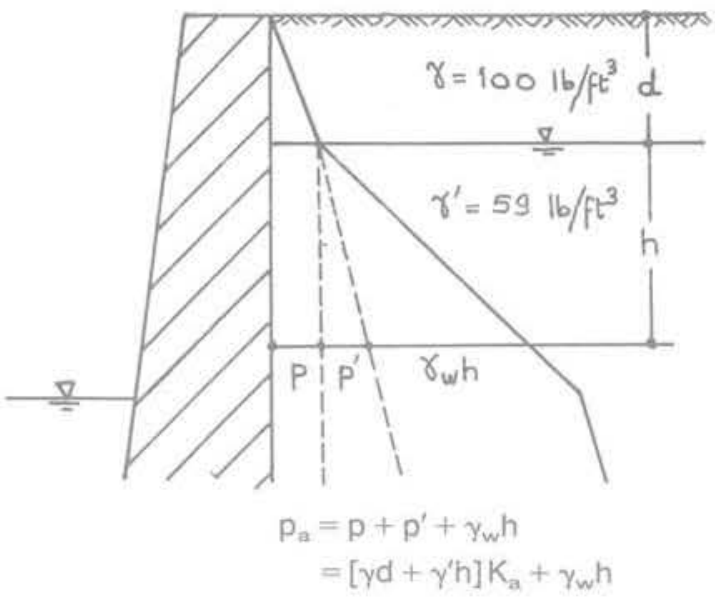

Fig. 18 D'après Baker, 1881

somme de la pression hydrostatique et de la pression latérale du remblai avec son poids unitaire réduit par la poussée. II donne comme exemple un remblai d'une porosité de $35 \%$. Dans l'air celui-ci pèse 100 livres/pied cube, $\left(16 \mathrm{kN} / \mathrm{m}^{3}\right)$ mais sous l'eau la contrainte latérale sera celle correspondant à la charge submergée, soit 59 livres/pied cube. $\left(9,4 \mathrm{kN} / \mathrm{m}^{3}\right)$ Ceci est parfaitement correct, mais le même raisonnement n'aurait probablement pas pu être appliqué à de l'argile, qui est considérée comme tout à fait imperméable.

\section{Biographie}

- Sir Benjamin Baker (1840-1907), F. R. S. LL. D. Président de I'Institution des Ingénieurs Civils. II fit partie de l'équipe de Sir John Fowler et à partir de 1869 fut son principal adjoint pour la construction du Métropolitain. II devint plus tard I'associé de Sir John et, avec celui-ci, fit les plans de nombreuses extensions du Système des Transports souterrains de Londres et du Pont sur te Forth. II fut également, entre autres, Conseil pour des travaux d'irrigation en Egypte. (Min. Proc. Inst. Civ. Eng. 170 (1907) pp. 377-383.)

\section{DARWIN - 1883}

\section{De la poussée horizontale d'une masse de} sable

Min. Proc. Inst. Civ. Eng. vol. 71 (1883) pp. 350-378.

Des essais ont été effectués sur une boîte de $22 \mathrm{~cm}$ de long et $30 \mathrm{~cm}$ de large dont l'un des côtés pivotait autour d'un axe horizontal et que l'on avait remplie de 18 à $34 \mathrm{~cm}$ de sable. Des essais faits avec soin sur le sable uniforme, $\mathrm{sec}$ et à grain fin, avaient donné un angle de frottement interne de $\beta_{n}=35 \pm 1^{\circ}$ et une densité à l'état meuble de 1,40. La face interne du panneau basculant avait une fine couche de sable collée à sa surface. Après avoir rempli la boîte jusqu'à une profondeur quelconque $\mathrm{H}$, la force qui maintenait le panneau fut réduite graduellement jusqu'à ce que le sable s'écoule, ce qui arrivait normalement lorsque le sommet du panneau s'était déplacé de $1 \mathrm{~mm}$ ou $1,5 \mathrm{~mm}$. 
Si le moment de renversement lors d'entrée en plasticité est $\mathrm{M}$, et si la composante horizontale de la poussée est $\mathrm{P} \cos \delta$ et en admettant que le centre de poussée est situé au tiers inférieur de l'écran on a :

et, posant

$$
\begin{gathered}
M=\frac{H}{3} P \cos \delta \\
P=\frac{1}{2} \gamma H^{2} K \\
M=\frac{1}{6} \gamma H^{3} K \cos \delta .
\end{gathered}
$$

on a

Dans les premières séries d'essais la boîte était remplie de sable meuble placé en couches horizontales. Les résultats montrèrent que $\mathrm{M}$ était vraiment proportionnel à $\mathrm{H}^{3}$ et que l'on avait $\mathrm{K} \cos \delta=0,18$ cette valeur étant la moyenne de 18 essais.

Après chaque essai le sable était complètement remanié avec un bâton et on martela les côtés de la boîte. Ainsi densifié le sable avait une densité de 1.55 et on avait $\mathrm{K} \cos \delta=0,132$. D'autres essais ont été faits avec du sable meuble déversé en couches inclinées et aussi avec la surface inclinée à $\pm \beta_{n}$.

Prenant $\Phi=\beta_{n}$, Darwin compara ses résultats avec la formule de Rankine et, admettant $\delta=\Phi$ avec la formule de Boussinesq de 1876 (voir $n^{\circ} 23$ ) et en tira une théorie légèrement inexacte élaborée sans connaître la solution de Poncelet et Scheffler. Pour la première série d'essais, aussi bien Boussinesq que la théorie du coin donnent pour $\mathrm{K} \cos \delta$ environ 0,2 , mais Rankine (avec $\delta=0$ si $\beta=0$ ) donne 0,27 .

Darwin fit divers essais pour déterminer l'effet du frottement latéral dans la boîte et dans ses calculs de $\mathrm{M}$, d'où celui de $\mathrm{K}$, il admet une tolérance à cet égard, d'ailleurs probablement insuffisante. Mais compte tenu de la précision susceptible d'être obtenue, la conclusion doit être que les théories de Boussinesq et de Coulomb (Iorsqu'elles sont correctement appliquées) donnent des résultats satisfaisants pour du sable meuble.

Par contre, pour du sable compact, il y a un écart notable. Darwin arrive tout près de la vérité lorsqu'il indique que l'angle de frottement interne n'est pas nécessairement le même que l'angle au repos. En particulier il se rend compte que dans du sable compacté il doit y avoir "mouvement " du sable dans lequel un grain après l'autre a un mouvement de rotation, adopte une structure plus ouverte et fait que la masse entière occupe un volume supérieur. De plus, il indique que cet effet se produit presque certainement le long de certaines surfaces ou de régions étroites, lesquelles finalement deviennent le siège du glissement. Et ceci le conduit à conclure que la théorie du "coin " est physiquement préférable aux théories qui traitent le sable comme une masse continue.

Nous avons ici une appréciation claire de la dilatance et de la formation ultérieure de zones de cisaillement. Le phénomène de dilatance a été ultérieurement démontré et désigné par Osborne Reynolds ( $n^{\circ} 26$ ).

Darwin résume en disant : " qu'aucune masse de sable ne peut être réunie sans qu'il y ait eu un mode opératoire et que ce mode opératoire déterminera la nature de son équilibre limite ". Ce point, indique-t-il. avait été entrevu par feu le professeur Clerk Maxwell dans des discussions alors que lui (Darwin) commençait ses essais. Ceux-ci, peut-on ajouter, furent exécutés en 1877 et le Mémoire de Baker l'engagea à faire une publication du fait qu'il apparaissait "une certaine insuffisance de résultats expérimentaux" pour vérifier l'exactitude des théories de pression de terrain.

II considère que "l'élément historique" y compris la dilatance, "poussent essentiellement au traitement mathématique ". Mais Boussinesq, dans une "Note sur le Mémoire de $\mathrm{Mr}$ G. H. Darwin sur la poussée horizontale d'une masse de sablen (Min. Proc. Inst. Civ. Eng. vol. 72 (1883) pp. 262-271), comprend parfaitement que l'angle de frottement interne $\Phi$ sera en général supérieur à l'angle de frottement dans les couches supérieures d'une pente obtenue par déversement (c'est-à-dire l'angle au repos) et il indique que $\Phi$ peut être recalculé à partir des résultats des essais (par exemple dans la seconde série, $\Phi=43^{\circ}$, à partir de l'équation 23 (2)) Boussinesq suggère aussi qu'une valeur approximative de $\Phi$ peut être déterminée directement en plaçant le sable dans une boîte à fond rugueux, quel que soit son état de compaction et en penchant la boîte jusqu'à ce que commence le mouvement du sable.

Note: Le Mémoire de Darwin est d'une importance exceptionnelle en mécanique des sols pure Mais ses essais n'étaient pas toujours bien prévus pour la détermination exacte de la pression de terrain, du frottement de la paroi, etc. Les essais classiques à cette époque étaient ceux de Müller-Breslau exposés dans son ouvrage «Pressions de terrains et murs de soutènement » Stuttgart, 1906.

\section{Biographies}

- Sir George Howard Darwin (1845-1912), F.R.S., commença ses travaux scientifiques comme gradué du Trinity College, Cambridge, et devint «Plumian " professeur d'astronomie et de philosophie expérimentale en 1883. II était surtout une autorité en mathématiques appliquées, travaillant surtout en géophysique et dynamique cosmologique. (Dictionnaire de Biographie Scientifique, 1971.)

- Heinrich Franz Bernhard Muller-Breslau (1851-1925), professeur d'études de ponts à Hanovre et depuis 1888 professeur de génie des structures à Charlottenburg; il est considéré comme le fondateur de l'école moderne sur ce sujet en Allemagne. (Dictionnaire de Biographie Scientifique, 1974.)

\section{REYNOLDS - 1885-1887}

a) De la dilatance des milieux constitués par des particules rigides en contact; avec des commentaires expérimentaux

Phil. Mag. 5th Ser, vol. 20 (1885 pp. 469-41

b) Expériences montrant la dilatance, une propriété de matériaux granuleux, en relation possible avec la gravitation

Proc. Roy, Institution vol. 11 (1887) pp. 354363.

Reynolds commence son Mémoire de 1885 par trois affirmations générales :

1 La dilatance, un terme introduit pour la première fois, est le changement de volume résultant du changement des positions relatives des grains d'une masse de matériaux meubles. Un exemple simple est la dilatation d'un groupe de particules sphériques 
passant d'une disposition tétrahédrale à une disposition cubique; ce qui correspond à une augmentation de volume de $30 \%$.

2 La dilatance dans une masse granuleuse étant essentiellement un phénomène géométrique, sera largement sinon complètement indépendante du frottement intergranulaire.

3 On peut peut-être admettre que les contraintes principales lors de la rupture sont liées par la relation de Rankine

$$
\frac{\sigma_{3}}{\sigma_{1}}=\frac{1-\sin \Phi}{1+\sin \Phi}
$$

où $\Phi$ est l'angle de talus naturel. Mais, dit Reynolds, on ne peut pas admettre que $\operatorname{tg} \Phi$ dépende du frottement réel entre les particules, la valeur de $\Phi$ étant fonction de leur arrangement. Ce troisième point, présenté comme résultat des réfiexions de Reynolds sur la dilatance, est particulièrement intéressant. En effet. jusque tout récemment, c'est plutôt le contraire qui était généralement admis, notamment que $\Phi$ dépendait largement du frottement intergranulaire. Mais la conception de Reynolds était correcte. Dans un matériau sans cohésion, $\Phi$ est presque exclusivement fonction de la forme et de la disposition des particules et presque pas du coefficient de frottement entre les grains, ce qu'à démontré dans une série d'essais très remarquables A. E. Skinner, essais qui ont fait l'objet d'une " note sur l'influence du frottement intergranulaire sur la résistance au cisaillement d'un ensemble quelconque de particules sphériques ". Géotechnique vol. 19 (1969) pp. 150-157

Reynolds décrit ensuite quelques expériences sur de la grenaille de plomb qui démontrent clairement les effets de la dilatance. Mais je vais passer de suite à son second Mémoire qui traite d'essais sur le sable. Ces essais ont été exécutés lors d'une conférence à la Royal Institution en février 1886 et deux d'entre eux sont classiques.

Dans le premier, un sac de caoutchouc mince contenant 6 pintes $(3,4$ litres) de sable compact dont les interstices sont remplis d'eau, est relié à un long tube de verre. En comprimant le sac entre deux planches, on y introduit de l'eau (on ne l'exprime pas comme ce serait le cas avec une éponge) et lorsque la dilatance est maxima le volume a augmenté de $15 \%$. En cet état la dilatance a amené ce matériau à sa densité minimale compte tenu des conditions de l'essai, bien qu'un chargement ultérieur tende à causer des contractions et expansions alternatives sur une moindre échelle.

Dans la seconde expérience, le sac est de nouveau rempli de sable dense, complètement saturé, mais cette fois le col du sac est fermé par un manomètre à mercure. Au moment du chargement le mercure monte du côté connecté au sac. Le sable devient presque rigide puisqu'aucune modification de volume ne peut se produire (sauf dans une faible mesure du fait du manomètre) et, avec une charge de 200 livres (90 kg) sur les planches, la pression dans le sac est inférieure de 27 pouces $(69 \mathrm{~cm}) 2092$ et mercure à celle de l'atmosphère. Si on ouvre le col pour permettre une entrée d'eau, le sac se déforme même sous une faible charge, et change de forme, mais ce changement s'arrête immédiatement quand on coupe l'arrivée d'eau.

On voit que les essais de Reynolds demontrent directement qu'un sable dense soumis à des contraintes de cisaillement présente : a) une augmentation de volume due à la dilatance dans un système ouvert (drainé),

b) une réduction de la pression interstitielle dans un système fermé (non drainé) accompagnée par une augmentation de la résistance.

II explique ensuite la résistance temporaire du sable de plage humide lorsqu'on marche dessus, ceci résultant de la dilatance et des tensions capillaires et le ramollissement du sable, si on reste en place, du fait que de l'eau y pénètre, venant des zones adjacentes et réduit les tensions. II explique enfin le peu de résistance de ce sable, en toutes circonstances, lorsqu'il est sec ou complètement submergé. II termine par des considérations éminemment spéculatives quant au rôle de la dilatance dans la théorie de la gravitation.

Le Professeur P. W. Rowe (1969) a poursuivi cette discussion et présenté quelques nouvelles expériences.

\section{Biographie}

- Osborne Reynolds (1842-1912), F.R.S. et titulaire de la Médaille Royale; ayant reçu une formation d'ingénieur, il enseigna les mathématiques à Cambridge et, après une courte période d'application devint Professeur de Génie Civil à Manchester en 1868. Là il exécuta des travaux particulièrement remarquables, en particulier en hydraulique et en lubrification. II prit sa retraite pour raison de santé, en 1905.

(Dictionnaire de Biographie Scientifique, 1975.)

\section{BOUSSINESQ - 1885}

\section{Application des potentiels à l'étude de l'équilibre et du mouvement des solides élastiques \\ Paris, Gauthier-Villars, 1885.}

L'ensemble de ce Traité fameux a été examiné en détails par Todhunter et Pearson (1893) et les conclusions importantes pour la mécanique des sols ont été résumées par Terzaghi (1943). Je rappellerai ici que pour la première fois Boussinesq

1 donne les contraintes et les déformations en un point quelconque à l'intérieur et sur la surface d'un solide élastique semi-infini, dues à une charge verticale ponctuelle en sa surface (horizontale),

2 dans un chapitre intitulé : "Calcul des dépressions que produisent à la surface d'un sol horizontal ... des pressions extérieures normales ", il donne la valeur moyenne, le tassement au centre et sur les bords, d'une aire circulaire chargée pour une distribution de pressions uniforme, parabolique, et parabolique inversée,

3 dans le même chapitre, il calcule le tassement d'une aire circulaire rigide et la distribution correspondante des contrepressions.

\section{RICHARDSON - 1908}

Les lignes d'écoulement de l'eau dans les sols saturés

Scientific Proc. Roy. Dublin Soc. vol. 11 (1908) pp. 295-316. 
S'inspirant du Traité élémentaire sur l'électricité de Clerk Maxwell (Oxford, 1881), Richardson développe la méthode par essai et erreur pour résoudre les réseaux d'écoulement à deux dimensions. II présenta un Mémoire à la Société de Physique en novembre 1907 sur "une méthode graphique de détermination des réseaux et des équipotentielles " (publié dans Phil. Mag. vol. 15 (1908) pp. 237-269), et complété par celui de Dublin qui fut imprimé en mai 1908.

Dans ce dernier il applique sa méthode à la détermination de la relation entre l'espacement des tranchées de drainage et la hauteur à laquelle l'eau de saturation va s'élever pour une pluviométrie donnée et une perméabilité connue. Les solutions sont appliquées au problème du drainage de masses de tourbe. La figure 19 montre un de ces réseaux soigneusement construit.

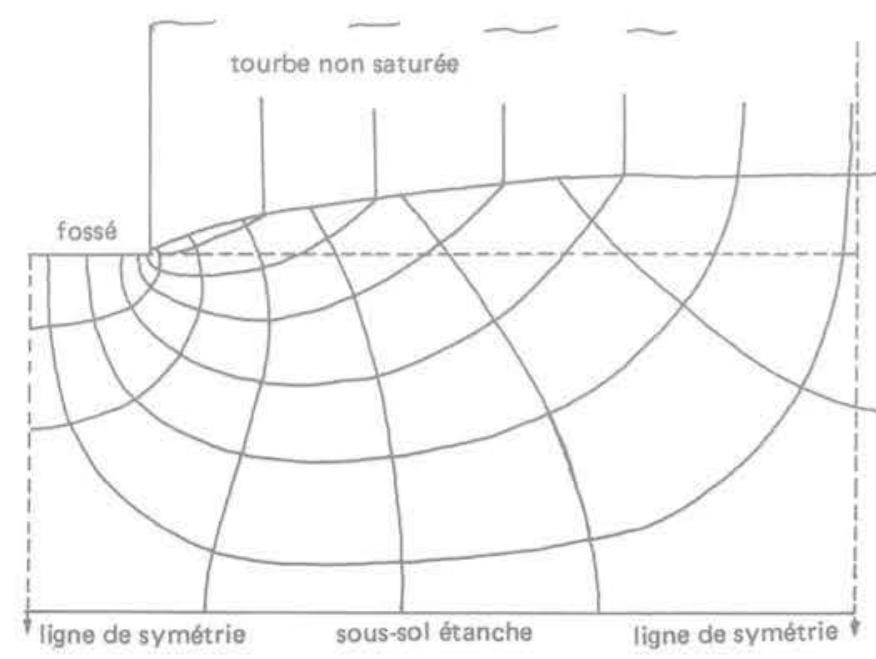

Fig. 19

Richardson traite très complètement des principes du calcul et des conditions aux limites sur une surface libre et de la technique du tracé des réseaux.

Le premier emploi de réseaux à mailles carrées comme procédé graphique de détermination de courant dans les problèmes d'hydraulique pure est attribué par Rouse et Ince (1957) à Franz Prasil de I'E.T.H. de Zurich dans son Hydrodynamique Technique (Berlin, 1913). Mais l'agrément général de cette technique et spécialement son développement dans les études d'infiltrations par Terzaghi et Casagrande, émane de I'Hydraulique de Forchheimer (1'éd. Leipzig. 1914).

\section{Biographies}

- Lewis Fry Richardson (1881-1953), F.R.S. poursuivit son travail sur les réseaux d'écoulement en imaginant en 1910 la méthode des différences finies pour résoudre les équations différentielles de Laplace en connaissant les conditions aux limites. Il travailla en météorologie de 1907 à 1920, sauf pendant son temps de service dans l'Ambulance des Amis (1916-1918). Ultérieurement, il a eu la charge du département de physique au Westminster College et de 1929 à 1940 a été Principal du Collège Technique de Paisley. (Roy. Soc. Obits, 9 (1954) 217-235.)
- Philipp Forchheimer (1852-1933), Professeur d'hydraulique à Aix-la-Chapelle et ensuite à Graz (Rouse et Ince, 1957). Pendantla Première Guerre mondiale il organisa la formation des ingénieurs à Istambul et eut l'initiative en 1916 d'inviter Terzaghi, qu'il avait connu comme étudiant à Graz, à son premier poste d'enseignant, à I'Université Technique d'Istambul. (Casagrande, Bjerrum et al, 1960).

\section{RÉSAL - 1910}

\section{Poussée des terres - deuxième Partie:} Théorie des terres cohérentes.

Paris, Béranger, 1910.

Résal admet avec Rankine que les argiles peuvent perdre leur cohésion à la suite de séchages et humidifications alternées, ainsi que du fait du gel et dégel, mais il indique que ces effets sont limités à la zone étroite des variations saisonnières. II est également possible, dit-il, que la cohésion disparaisse à de plus grandes profondeurs, si une argile est soumise à une pression interstitielle hydrostatique ou si elle contient des vides ou des fissures que l'eau peut facilement pénétrer. Toutefois, en pratique, comme on prend soin de compacter convenablement les remblais, et de drainer les tranchées, il doit y avoir beaucoup de cas où la cohésion peut être considérée comme un élément permanent de la résistance.

C'est pourquoi il est nécessaire de revenir au critère de rupture de Coulomb et d'obtenir des renseignements précis sur c et $\Phi$ dans les argiles, de préférence en mesurant la résistance au cisaillement sous différentes pressions normales. Mais en l'absence de renseignements de cette nature, Résal prend $\gamma=1.8 \mathrm{t} / \mathrm{m}^{3}$ $\left(18 \mathrm{kN} / \mathrm{m}^{3}\right), \mathrm{c}=3,6 \mathrm{t} / \mathrm{m}^{2}(36 \mathrm{kPa})$ et $\Phi=15^{\circ}$ comme valeurs pour des exemples traités quantitativement. Dans une contribution importante à la mécanique des sols classique il entreprend l'examen de quatre questions fondamentales.

\section{Analyse du champ de contraintes}

Tout d'abord il développe l'analyse du champ de contraintes de manière à inclure les sols cohérents. On trouvera le détail dans Terzaghi (1943), mais ici nous pouvons noter:

1 Dans le cas de la poussée la traction s'étend jusqu'à la profondeur

$$
\mathrm{z}_{\mathrm{o}}=\frac{2 \mathrm{c}}{\gamma} \operatorname{tg}\left(\frac{\pi}{4}+\frac{\Phi}{2}\right)=\frac{2 \mathrm{c}}{\gamma} \frac{\cos \Phi}{1-\sin \Phi}
$$

et cette profondeur est indépendante de la pente $\beta$.

2 Pour $\beta=0$ les lignes de glissement sont rectilignes et coïncident avec la solution de Rankine à toutes profondeurs en butée et à des profondeurs supérieures à $z_{0}$ en poussée.

3 Pour $\beta>0$ les lignes sont courbes, et si $\beta>\Phi$ les lignes de poussée comme celles de butée deviennent tangentes à une ligne inclinée à l'angle $\beta$ et à la profondeur

$$
z_{1}=\frac{c}{\gamma} \frac{\cos \Phi}{\sin (\beta-\Phi) \cos \beta}
$$

4 Dans la zone de traction, les lignes de glissement se confondent avec les lignes de rupture par traction qui sont normales à la surface. 


\section{Tranchée verticale}

5 La solution de Coulomb pour la hauteur critique d'une tranchée verticale dans un terrain horizontal

$$
H_{c}=\frac{4 c}{\gamma} \frac{\cos \Phi}{1-\sin \Phi}
$$

implique que le sol peut supporter des contraintes de traction, c'est-à-dire qu'il n'y a pas de fissures de traction. Ceci n'est pas confirmé par l'expérience, et pour dèterminer une limite plus basse, plus raisonnable dans la pratique, Résal admet que le sol ne peut supporter aucune traction. II trouve dans ce cas la valeur suivante pour la hauteur critique

$$
\mathrm{H}_{0}=\frac{2 \mathrm{c}}{\gamma} \frac{\cos \Phi}{1-\sin \Phi} \text {. }
$$

6 La même hypothèse conduit à la conclusion que les fissures de traction vont pénétrer jusqu'à la profondeur $z_{0}=H_{0}$. Une plaque verticale mince se rompra si la tranchée est entaillée plus profondément. Avec les paramètres indiqués ci-dessus

$$
\mathrm{H}_{\mathrm{o}}=5,2 \mathrm{~m} \text {. }
$$

\section{Stabilité des pentes}

7 L'équation (3) s'applique si $\beta=90^{\circ}$ et, à l'autre limite, la pente peut tenir sur une hauteur infinie si $\beta=\Phi$, Pour les cas intermédiaires, Résal obtient une solution approchée

$$
H=\frac{2 c}{\gamma} \frac{\sin \beta \cos \Phi}{1-\cos (\beta-\Phi)}
$$

basée sur une surface de glissement qui s'amorce au pied d'une fissure de traction de profondeur $z_{0}$ juste en arrière de la crête, devient tangente à un plan incliné à $\beta$ et situé à une profondeur $z_{1}$ et s'incurve en passant par le pied de la pente, où elle fait un angle de $\left(\frac{\pi}{4}-\frac{\Phi}{2}\right)$ avec la face.

Dans le tableau 6 les valeurs du facteur de stabilité données par l'équation (4) sont comparées à celles calculées par la méthode de l'arc circulaire (Taylor. 1948) auxquelles j'ai appliqué une correction approximative tenant compte de l'effet des fissures de traction. comme l'a suggéré Taylor lui-même.

Tableau 6 .

Valeurs de $\gamma \mathrm{H} / \mathrm{c}$ pour $\Phi=15^{\circ}$

\begin{tabular}{|c|r|r|r|r|}
\hline$\beta$ & $90^{\circ}$ & $60^{\circ}$ & $45^{\circ}$ & $30^{\circ}$ \\
\hline Résal & 2,6 & 5,7 & 10,2 & 28 \\
Taylor & 2,6 & 6,5 & 10,5 & 21 \\
\hline
\end{tabular}

\section{Murs de soutènement}

8 Résal traîte le cas général de la poussée sur un mur incliné, avec frottement sur le mur et terrain incliné. Pour la simplicité, cependant, nous considérons un mur vertical avec $\delta=0$ et remblai horizontal. La solution standard est alors

$$
\mathrm{p}_{\mathrm{a}}=\gamma \mathrm{z} \operatorname{tg}^{2} \varepsilon-2 \mathrm{c} \operatorname{tg} \varepsilon
$$

où $\varepsilon=\frac{\pi}{4}-\frac{\Phi}{2}$. Mais pour $z<z_{0}$ cette équation donne des valeurs négatives de $p_{a}$ inadmissibles dans un sol dépourvu de résistance à la traction. C'est dire que $\mathrm{p}_{\mathrm{a}}$ doit être pris égal à zéro dans la zone de traction, et que la pression totale sera

$$
p_{a}=\int_{z_{0}}^{H} p_{a} d z=\frac{1}{2} \gamma\left(H-z_{0}\right)^{2} \operatorname{tg}^{2} \varepsilon .
$$

Cette expression correspond exactement au résultat obtenu par la méthode de l'analyse du coin lorsque les fissures de traction s'étendent jusqu'à la profondeur $z_{0}$. Inversement, si on intègre $p_{a} d z$ de $z=0$ à $z=H$ cela montre que le résultat correspond exactement à l'analyse avec le coin de Coulomb qui implique qu'il n'y a pas de fissure de traction (fig. 20).

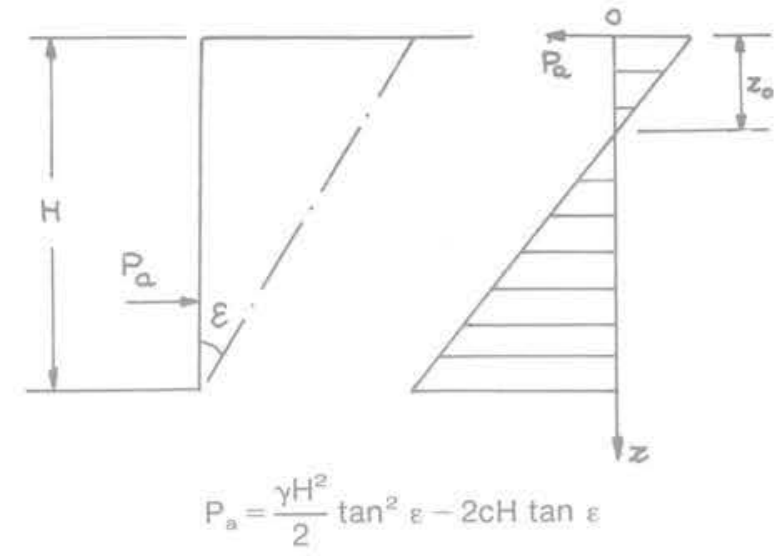

Coulomb

$$
E=(45-\phi / 2) \quad \delta=0 \quad z_{0}=\frac{2 c}{\gamma} \tan (45+\phi / 2)
$$

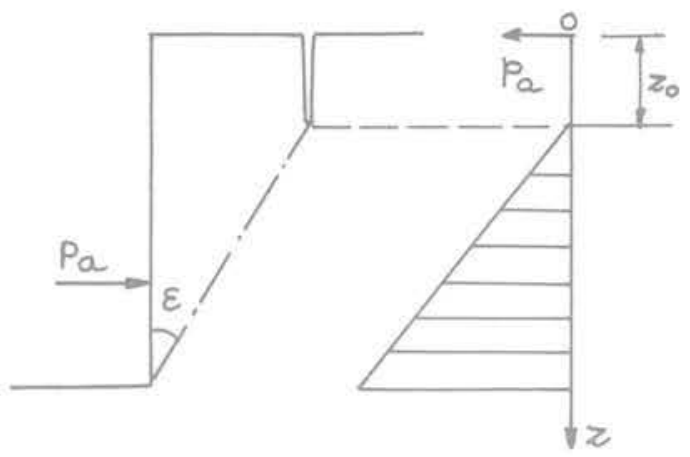

$$
P_{a}=\frac{\gamma}{2}\left(H-z_{0}\right)^{2} \tan ^{2} \varepsilon
$$

Résal

Fig. 20 Poussée dans l'argile

L'équation (5) a été donnée pour la première fois par Résal. Mais elle avait été calculée indépendamment par le Professeur Cain, qui explique très clairement les points qui font l'objet du précédent paragraphe, dans ses "Essais sur les murs de soutènement et les pressions sur les tunnels ".

Trans. Am. Soc. Civ. Eng. vol. 72 - (1911) pp. 403-448.

9 Résal traite aussi de la butée et analyse la stabilité contre le glissement, mais ces points ne comportent d'autre commentaire que le fait qu'il ne tient pas compte du frottement sur le mur dans ses calculs de butée. 
10 Lorsque le livre était sur le point d'être envoyé à l'imprimerie, il ajouta un résumé des résultats d'essais de cisaillement obtenus en juillet et août 1910 par Frontard sur le remblai d'argile de la digue en terre de Charmes en Haute-Marne (voir $n^{\circ} 30$ ). Les essais, faits à l'instigation de Résal, confirmèrent le critère de Coulomb $\mathrm{s}=\mathrm{c}+\sigma \operatorname{tg} \Phi$ et montrèrent typiquement $\mathrm{c}=2 \mathrm{t} / \mathrm{m}^{2} \quad(20 \mathrm{kPa})$ et $\Phi=8^{\circ}$. Comme ses essais correspondaient approximativement à l'état non drainé, une valeur de $8^{\circ}$ n'était pas surprenante, mais à l'époque elle fut regardée avec quelque étonnement. (Voir les remarques finales du Professeur Cain dans la discussion de ce Mémoire, le livre de Résal étant dans l'intervalle arrivé aux États-Unis.)

\section{Biographies}

- Jean Résal (1854-1919), bien connu pour ses études de ponts, fut professeur de résistance des matériaux à l'Ecole des Ponts et Chaussées depuis 1896 (Annales des Ponts et Chaussées $10^{e}$ série 55 (1920) 147-168).

- William Cain (1847-1930), après avoir travaillé dans les chemins de fer, aborda le professorat et de 1888 à 1920 enseigna le génie civil à I'université de la Caroline du Nord (Trans. Am. Soc. Civ. Eng. 95 (1931) 1467-1472).

\section{Notes sur les essais de résistance au cisaillement par Frontard et Bell, et la Période 1910-1926.}

Les essais de Jean Frontard en 1910 (voir $n^{\circ} 29$ ) sont décrits dans son article (Notice sur l'accident de la digue de Charmes) Annales des Ponts et Chaussées $9^{e}$ série vol. 22 (1914) pp. 173-280. Un an après, Arthur Langtry Bell construisit la première boîte de cisaillement pratique et en 1911-12 fit des essais sur 8 argiles, dont deux à l'état d'échantillons intacts. Comme ceux de Frontard, ces essais étaient assez rapides, répondant à peu près à la condition "non drainés" et, comme les argiles étaient complètement ou presque tout à fait saturées, les valeurs de $\Phi$ ont tendu à être inférieures aux $8^{\circ}$ obtenus par Frontard. Elles approchèrent même de zéro pour les plus molles.

Conforté par ces résultats, Bell les utilisa pour étudier un grand mur de dock à Rosyth et, en 1913, vérifia la pression calculée au moyen d'observations directes avec des cellules. Son mémoire, "Pression latérale, résistance de l'argile et pouvoir portant des fondations sur l'argile ", fut publié dans Min. Proc. Inst. Civ. Eng. vol. 199 (1915) pp. 233-272. II constitue le début de la mécanique des sols moderne.

Les essais de Frontard et le travail important de Bell à Rosyth ont fait l'objet d'une importante discussion par Skempton (1958) et ne seront pas repris ici. Car en réalité ils appartiennent à la phase d'initiation à une période nouvelle qui déborde le cadre de la présente conférence.

Cette nouvelle phase d'activité comprend les recherches d'Atterberg sur la plasticité des sols et la dimension des particules (son Mémoire sur les limites de liquidité et de plasticité parut en 1911), l'introduction de l'analyse du cercle de glissement en 1916 (voir l'excellent exposé historique de Petterson en '1955) et le travail de laboratoire et de chantier exécuté entre 1914 et 1920 par la Commision de Géotechnique des
Chemins de fer Suédois (voir Bjerrum and Flodin, 1960).

Ensuite ce furent les expériences fondamentales de Terzaghi publiées entre 1921 et 1924, d'où sortit le principe de la contrainte effective (Skempson, 1960), et son ouvrage monumental Erdbaumechanik (Vienne, 1925), suivi la même année par les articles de I'Engineering News Record auxquels il est fait allusion dans l'introduction. Ceux-ci parurent en librairie en 1926, sous le titre "Principes de mécanique des sols", exactement 150 ans après la publication de l'ouvrage de Coulomb.

Cette conférence a été prononcée par le Professeur A. W. Skempton au cours du $7^{\circ}$ Congrès Européen de Mécanique des Sols et des Travaux de Fondations à Brighton. conformément à la tradition anglaise un vote de remerciements a été soumis à l'Assemblée. C'est à Jean Kerisel qu'est revenu l'honneur de le proposer. II a été accepté chaleureusement et à l'unanimité.

\section{Références bibliographiques}

BJERRUM L., CASAGRANDE A., PECKR. B. and SKEMPTON A. W. (1960). From Theory to Practice in Soil Mechanics. New York : Wiley.

BJERRUM L. and FLODIN N. (1960). « The development of soil mechanics in Sweden." Geotechnique 10, 1-18.

BOUSSINESQ J. (1885). Application des Potentie/s, réimpression avec une préface de $\mathrm{A}$. Caquot. Paris : Blanchard, 1969.

FELD J. (1923). « The accurate experimental determination of lateral earth pressure, together with a résumé or previous experiments." Trans. Am. Soc. Civ. Eng. 86, 1448-1505.

FLODIN N. and BROMS B. B. (1977). " Historical development of civil engineering in soft clay. " International Symposium on Soft Clay (Bangkok).

GLOSSOP R. (1960). « The invention and development of injection processes. Part I : 1802-1850. " Geotechnique $10,91-100$.

GLOSSOP R. R. (1961). «The invention and development of injection processes, Part II: 1850-1960, " Geotechnique 255-279.

GLOSSOP R (1976). " The invention and early use of compressed air to exclude water from shafts and tunnel during construction. " Geotechnique 26, 253280.

HALL H. P. (1954). «A historical review of investigations of seepage toward wells'. " Journ. Boston Soc. Civ. Eng. 41, 251-311.

HEYMAN J. (1972). Coulomb's Memoir on Statics. Cambridge University Press.

KERISEL J. (1956). « Historique de la mécanique des sols en France jusqu'au $20^{\circ}$ siècle. " Geotechnique 6 , 151-166.

MAYNIEL J. H. (1808). Traité expérimental, analytique et pratique de la Poussée des Terres et des Murs de Revêtement. Paris : Colas.

PECK R. B. (1948). History of Building Foundations in Chicago. Univ. Illinois Eng. Expt. Station, Bulletin $N^{\circ} 373$.

PETTERSIN K. E. (1955). « The early history of circular sliding surfaces." Geotechnique 5, 275-296. 
ROUSE H. and INCE S. (1957). History of Hydraulics. lowa State University.

ROWE P. W. P.W. (1969). "Osborne Reynolds and dilatancy. " Geotechnique 19, 19, 1-5.

SCHRIEVER W. R. (1956). Landslides in Clays by Alexander Collin, 1846. Translation, with a memoir on Collin by A. W. Skempton. Toronto University Press.

SKEMPTON A. W. (1958). « Arthur Langtry Bell (18741956) and his contribution to soil mechanics." Geotechnique 8, 143-157.

SKEMPTON (1960). \& Terzaghi's discovery of effective stress , in Bjerrum et al. pp. 42-53.
TAYLOR D. W. (1948). Fundamentals of soil mechanics. New York : Wiley.

TERZAGHI K. (1925). "Principles of soil mechanics" Eng. News. Record 95. Eight articles between pp. 742 and 1068 . Published as a book with the same title. New York : McGraw-Hill, 1926.

TERZAGHI K. (1943). Theoretical Soil Mechanics. New York : Wiley.

TIMOSHENKO S. P. (1953). History of Strength of Materials. New York: McGraw-Hill.

TODHUNTER I and PEARSON K. (1893). A History of the Theory of Elasticity and of the Strength of Materials. Cambridge University Press. 